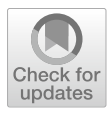

Cite as

Nano-Micro Lett.

(2021) $13: 136$

Received: 13 April 2021

Accepted: 11 May 2021

Published online: 7 June 2021

(C) The Author(s) 2021

\section{Engineering the Coordination Sphere of Isolated Active Sites to Explore the Intrinsic Activity in Single-Atom Catalysts}

\author{
Xin $\mathrm{Wu}^{1,2}$, Huabin Zhang ${ }^{2}{ }^{凶}$, Shouwei Zuo ${ }^{2}$, Juncai Dong ${ }^{3}$, Yang $\mathrm{Li}^{1}$, Jian Zhang ${ }^{1}$, \\ $\mathrm{Yu} \mathrm{Han}{ }^{4} \bowtie$
}

\title{
HIGHLIGHTS
}

- All the coordination engineering strategies, such as tuning the coordination species, the coordination number of the active centers, heteroatoms interactions within the support, synergetic interaction between neighboring metal monomers, and spatial microenvironment, have been summarized and discussed in detail.

- Various single-atom catalysts (SACs) with different coordination spheres in energy conversion driven by thermal, light and electric energy have been systematically reviewed.

- The current key challenges in SACs for energy conversion are pointed out, and some potential strategies/perspectives are proposed.

\begin{abstract}
Reducing the dimensions of metallic nanoparticles to isolated, single atom has attracted considerable attention in heterogeneous catalysis, because it significantly improves atomic utilization and often leads to distinct catalytic performance. Through extensive research, it has been recognized that the local coordination environment of single atoms has an important influence on their electronic structures and catalytic behaviors. In this review, we summarize a series of representative systems of single-atom catalysts, discussing their preparation, characterization, and structureproperty relationship, with an emphasis on the correlation between the coordination spheres of isolated reactive centers and their intrinsic catalytic activities. We also share our perspectives on the current challenges and future research promises in the development of single-atom catalysis. With this article, we aim to highlight the possibility of finely tuning the catalytic performances by engineering the coordination spheres of single-atom sites and provide new insights into the further development for this emerging research field.
\end{abstract}

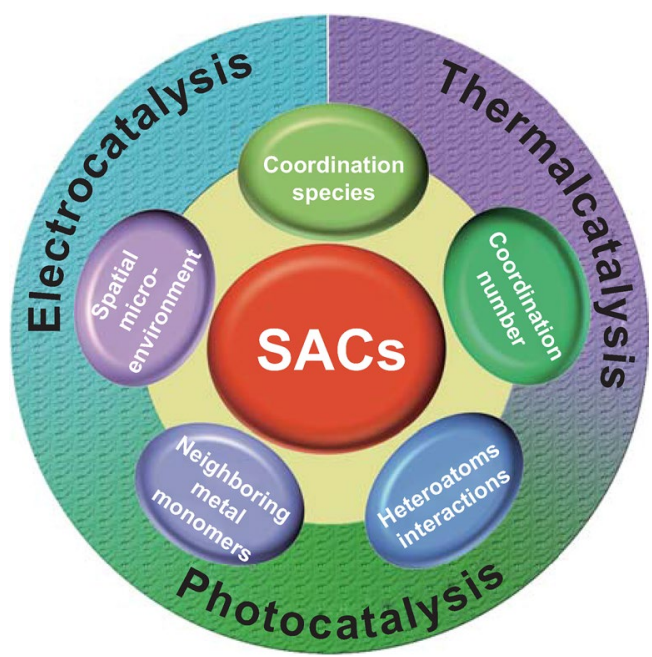

KEYWORDS Isolated atoms; Coordination sphere; Intrinsic activity; Single-atom catalysts

Huabin Zhang, Huabin.Zhang@kaust.edu.sa; Jian Zhang, Zhj@ jjirsm.ac.cn; Yu Han, Yu.Han@kaust.edu.sa

1 State Key Laboratory of Structural Chemistry, Fujian Institute of Research on the Structure of Matter, Chinese Academy of Sciences,

Fuzhou 350002, People's Republic of China

2 KAUST Catalysis Center, Physical Sciences and Engineering Division, King Abdullah University of Science and Technology, Thuwal 23955-6900, Saudi Arabia

3 Beijing Synchrotron Radiation Facility, Institute of High Energy Physics, Chinese Academy of Sciences, Beijing 100049, People's Republic of China

4 Advanced Membranes and Porous Materials Center, Physical Sciences and Engineering Division, King Abdullah University of Science and Technology, Thuwal 23955-6900, Saudi Arabia 


\section{Introduction}

Single-atom catalysts (SACs) composed of isolated metal atoms as the active sites are considered to bridge the gaps between the homogeneous and heterogeneous catalysts [1-10]. The concept of "single-atom catalysts" was firstly proposed by Zhang and colleagues in 2011. In their pioneering work, they demonstrated that the isolated, single Pt atoms stabilized on an iron oxide support have extraordinary activity for $\mathrm{CO}$ oxidation [11]. Subsequent studies indicated that in SACs, the atomic dispersion of catalytic centers with nearly identical configuration not only represents the extreme atomic utilization, but also offers enormous opportunities to elucidate the underlying catalytic mechanism at atomic level [12-15]. However, preparing SACs with atomic precision is challenging, due to the high surface energy and tedious synthetic procedures, which largely hinders their practical applications. Thus it is of vital significance to develop synthetic strategy to prepare SACs with robust and well-defined structures from both academic and industrial points of view [16].

The reactive centers in SACs are greatly modulated by the surrounding atoms via the strong electronic coupling [17-22]. Thus, regulating the coordination sphere of the reactive center will significantly influence the intrinsic activity and stability of SACs, promoting their rapid optimization to satisfy the requirements of targeted reactions [23, 24]. Tremendous research endeavors have been devoted to tuning the performance of isolated reactive centers via coordination engineering [25-28]. At present, several instructive articles have reviewed the SACs fabrication and coordination environment regulation. Most works merely focus on the metal-support interactions and reaction mechanisms for electrochemical or photocatalytic reactions, lacking a comprehensive and systematical understanding of strategies toward promoting various catalytic reactions based on SACs [29-32]. Recently, the extensive exploration of various modulation strategies for SACs extends their application in wider catalysis systems. Therefore, a timely summary is highly needed on this fast developing area.

Herein, we summarize the latest advances in modulating the coordination spheres of SACs and analyze the key structural characteristics those influence the intrinsic activity of isolated reactive sites (Fig. 1). We center our discussions on the direct structure-performance relationship of the isolated reactive centers and the underlying mechanisms for the particular reaction types. Through some examples, we demonstrate how the catalytic selectivity, intrinsic activity and stability of SACs can be optimized by engineering the coordination sphere of the reactive centers. In the last section, we outline the current challenges and possible solutions associated with SACs. We expect that this review will inspire a more comprehensive understanding and in-depth discussion on the SACsinvolved catalytic mechanisms, thus promoting the further development of this emerging research area.

\section{Rational Designs of SACs}

The successful design of SACs requires the harmonization of multiple demands, such as high stability, uniform active sites, tunable electronic, geometric geometry and so on. However, it is still challenging to prevent the aggregation as the ultrahigh surface energy of isolated reactive centers. To address this critical issue, enormous attention has been focused on the stabilization and confinement of isolated metal atoms. In the current section, we will briefly introduce various strategies for producing SACs.

\subsection{Wet-chemistry Strategies}

Generally, the wet-chemistry strategies include co-precipitation, impregnation $[33,34]$ and photochemical strategy, etc. In the wet-chemistry routes, consecutive processes are combined. The metal species are first anchored on various supports, followed by drying and annealing. At last, SACs with homogeneously dispersed reactive centers can be obtained after further reduction or activation. Although the SACs prepared by these strategies usually faced up with some drawbacks, such as low loading contents and poor dispersion of single atoms, wet-chemistry strategies have been extensively adopted as effective and convenient methods to prepare SACs [35, 36]. Zhang and colleagues have successfully developed a series of SACs via coprecipitation method with the metal atoms ranging from $\mathrm{Pt}$, Ir to Au [11, 37-39]. When the aqueous solution of 


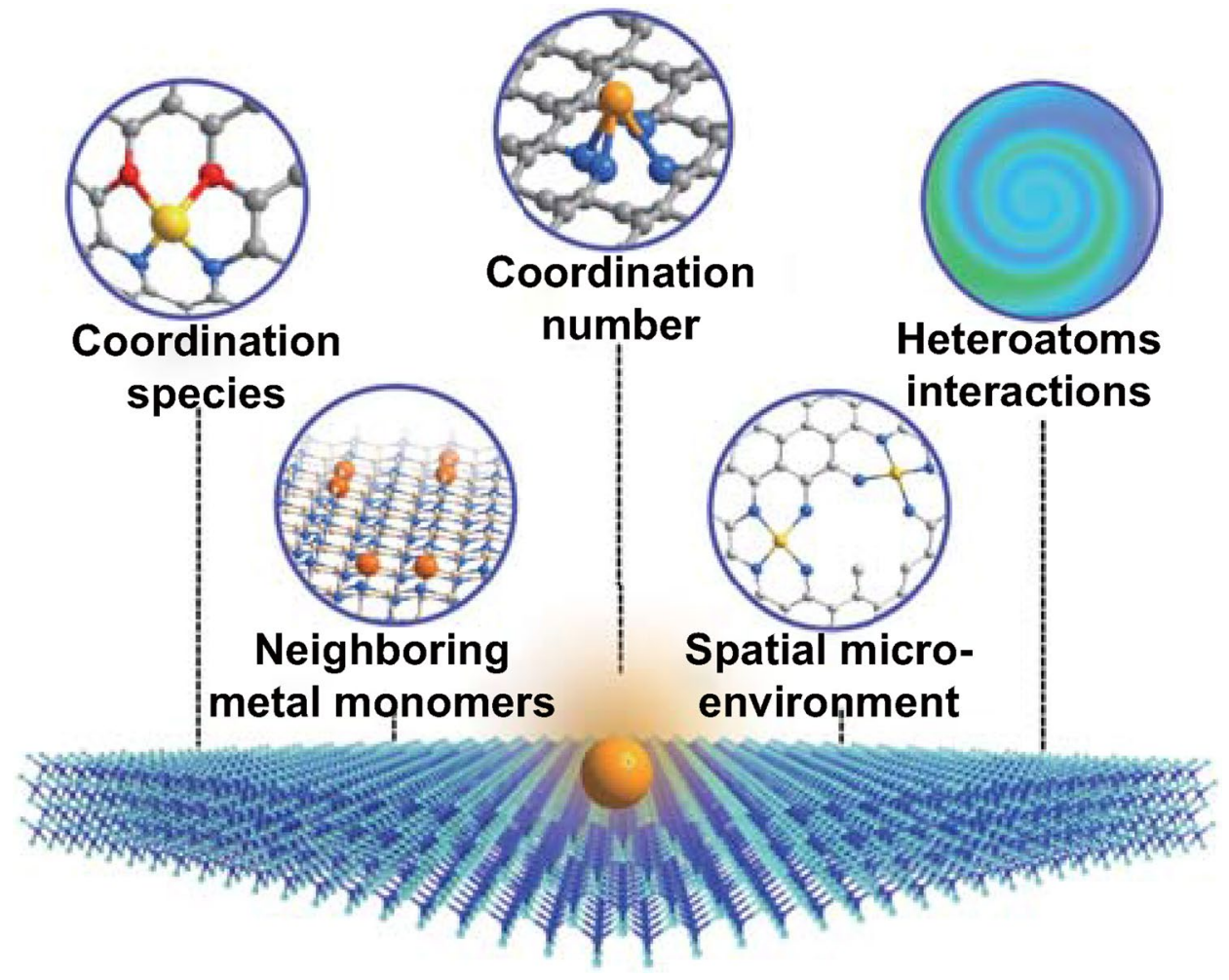

Fig. 1 Schematic illustration of the strategies to engineer coordination sphere of isolated active sites

chloroplatinic acid and ferric nitrate is mixed with sodium carbonate at a finely tailored reaction temperature and $\mathrm{pH}$ value, $\mathrm{Pt}$ species can be anchored on the defects of $\mathrm{FeO}_{\mathrm{x}}$. Calcination and reduction processes are further proceed to obtain the final Pt SACs.

Ag alloyed Pd SACs supported on silica gel $\left(\mathrm{AgPd} / \mathrm{SiO}_{2}\right)$ have been prepared by a simple impregnation method. [40] After impregnation of silica gel with $\mathrm{Pd}\left(\mathrm{NO}_{3}\right)_{2}$ and $\mathrm{AgNO}_{3}$ solutions, bimetallic $\mathrm{AgPd} / \mathrm{SiO}_{2}$ is obtained via calcination in air at $400{ }^{\circ} \mathrm{C}$ for $2 \mathrm{~h}$. In the reduction process, $\mathrm{Pd}$ atoms are isolated on the optimal $\mathrm{Pd} / \mathrm{Ag}$ surface ensembles. Zheng and coworkers have synthesized stable $\mathrm{Pd} / \mathrm{TiO}_{2}$ SACs with the Pd loading content of $1.5 \mathrm{wt} \%$ through a photochemical method (Fig. 2a-c) [41]. Ethylene glycolate decorated ultrathin $\mathrm{TiO}_{2}$ nanosheet is selected as the support, which can generate radicals under ultraviolet light to promote the removal of $\mathrm{Cl}^{-}$ligand from $\mathrm{Pd}$ sites in $\mathrm{H}_{2} \mathrm{PdCl}_{4}$ and form the isolated $\mathrm{Pd}$ atoms via $\mathrm{Pd}-\mathrm{O}$ bonds. The finely synthesized $\mathrm{Pd}_{1} / \mathrm{TiO}_{2}$ catalyst shows prominently high stability and activity in the hydrogenation of $\mathrm{C}=\mathrm{C}$ and $\mathrm{C}=\mathrm{O}$.

\subsection{Metal-organic Frameworks (MOFs)-Participated Approaches}

MOFs as functional porous materials have attracted enormous attention for many applications related to catalysis, gas adsorption and separation [42-46]. The diverse architectures and compositions make it possible to incorporate multifunctional catalytic centers in MOFs and tailor the configuration of SACs, which render them to be unsurpassed precursors for the generation of SACs [47-49]. More importantly, mechanistic insights into catalytic activity origin of SACs can be achieved through the precise control of the structures of SACs.

The MOF itself can be applied as the support for stabilizing the isolated reactive centers. Ye and colleagues have realized the dispersion of isolated Co active centers into the porphyrin unit of the MOF-525 [50]. The porphyrin units not only work as the anchoring site for isolating the reactive centers, but also can modularize the coordination geometry of the isolated Co centers. Directional 

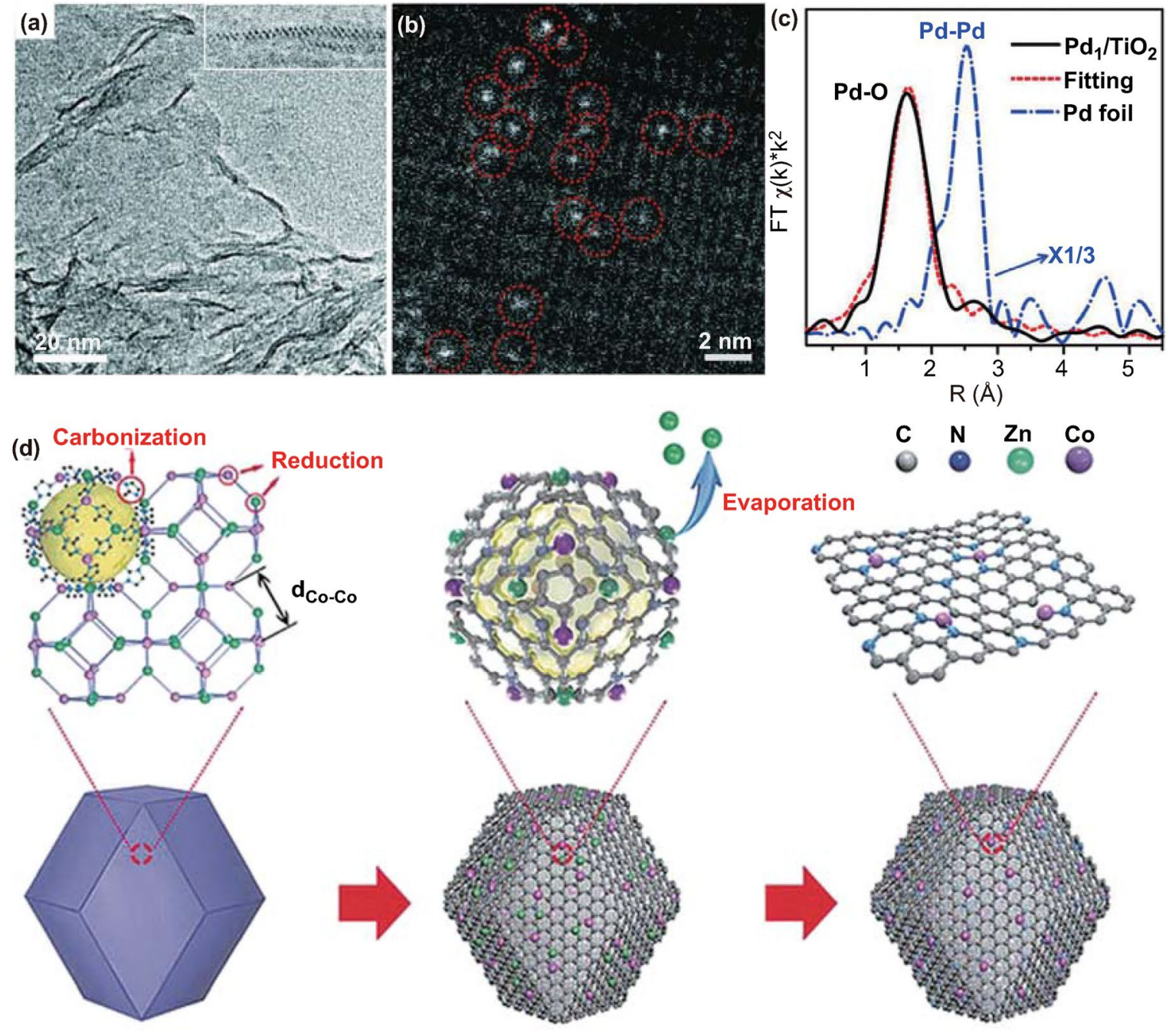

Evaporation

(e)

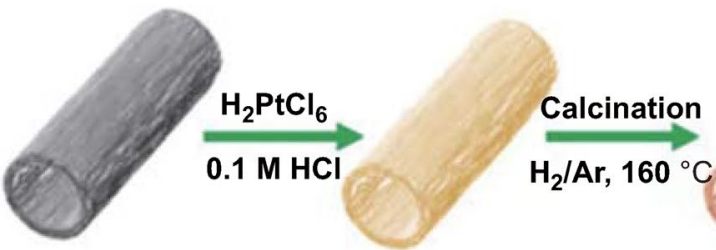
$\mathrm{Na}_{\mathrm{x}} \mathrm{H}_{2-\mathrm{x}} \mathrm{Ti}_{3} \mathrm{O}_{7}$ Pt-Na ${ }_{x} \mathrm{H}_{2-\mathrm{x}} \mathrm{Ti}_{3} \mathrm{O}_{7}$

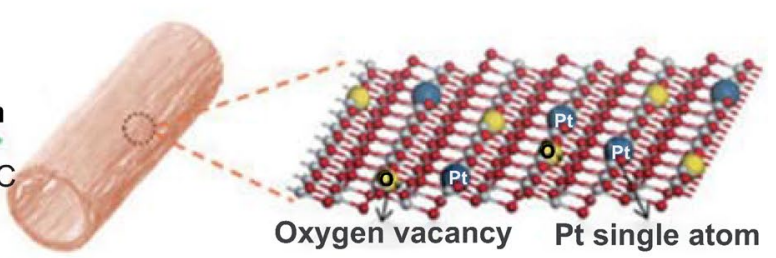

(f)
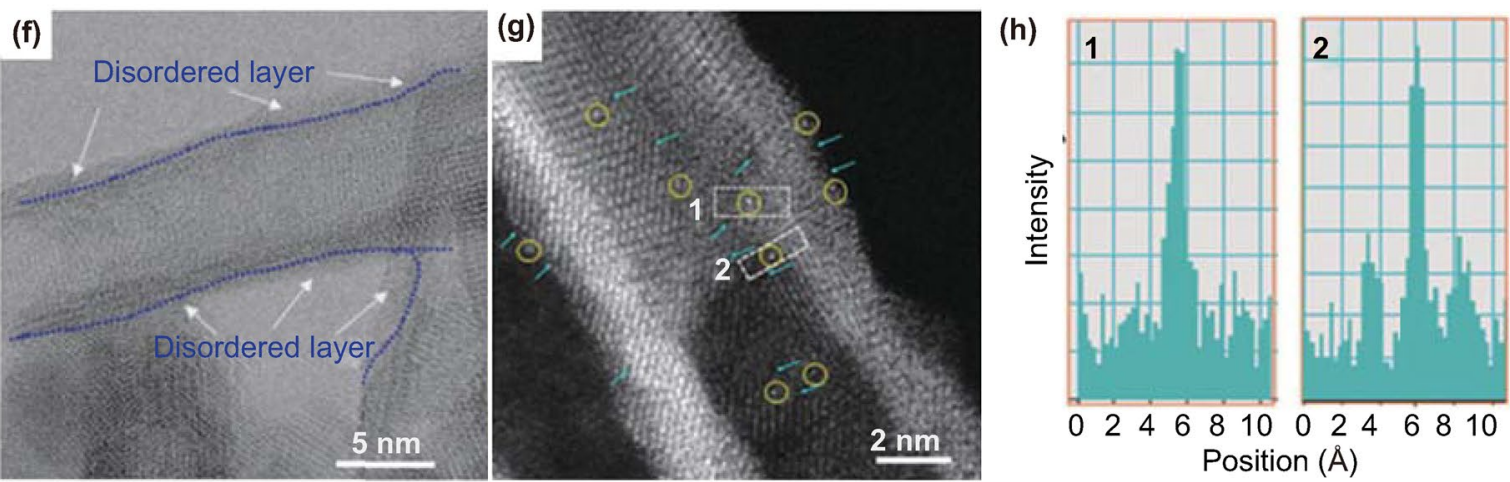
4Fig. 2 a Representative TEM image of $\mathrm{Pd}_{1} / \mathrm{TiO}_{2}$. The inset is an aberration-corrected STEM image for cross sections of ultrathin $\mathrm{TiO}_{2}(\mathrm{~B})$, showing that it is composed of only two layers of $\mathrm{Ti}$ atoms. b HAADF-STEM image of $\mathrm{Pd}_{1} / \mathrm{TiO}_{2}$. c FT-EXAFS spectra of $\mathrm{Pd}_{1} /$ $\mathrm{TiO}_{2}$ and bulk palladium foil at the Pd K-edge, showing the surrounding atoms adjacent to Pd atoms. Reproduced from Ref. [41] with permission. Copyright 2016, American Association for the Advancement of Science. d The formation of Co SAs/N-C. Reproduced from Ref. [54] with permission. Copyright 2016 Wiley-VCH Verlag GmbH \& Co. KGaA, Weinheim. e The preparation process of the $\mathrm{Pt}_{1} /$ def-TiO catalyst. $\mathbf{f}$ HRTEM image and $\mathbf{g}$ AC HAADF-STEM image of the $\mathrm{Pt}_{1} /$ def- $\mathrm{TiO}_{2}$ catalyst. The $\mathrm{Pt}$ atoms are marked by the yellow circles. The defective structure of the $\mathrm{TiO}_{2}$ support is marked by a blue arrow. $\mathbf{h}$ The intensity profiles obtained in regions 1 and 2 in $\mathbf{g}$. e-h Reproduced from Ref. [61] with permission. Copyright 2020 Wiley-VCH Verlag $\mathrm{GmbH} \& \mathrm{Co}$. KGaA, Weinheim

transfer of photo-generated electrons from porphyrin units to the isolated Co centers has been observed, realizing the supply of photo-generated electrons for the $\mathrm{CO}_{2}$ reduction over the isolated Co centers. Lin and colleagues have further applied MOFs to stabilize the low coordinated Ir species for methane borylation [51]. The isolated Ir atoms in the mono(phosphine)-Ir-based MOF ( $\mathrm{Zr}-\mathrm{P}_{1}$-Ir) adopt the distorted tetrahedral geometry and are coordinated by one chloride, one bidentate 1,5-cyclooctadiene, and one mono(phosphine) from the MOF ligand. Comparing with some other Ir catalysts, $\mathrm{Zr}$ - $\mathrm{P}_{1}$-Ir delivers more impressive catalytic performance in methane borylation to exclusively afford $\mathrm{CH}_{3}$ Bpin with a turnover number (TON) of 127 at $110{ }^{\circ} \mathrm{C}$. Furthermore, by utilizing the post-synthetic modification of MOFs, individual metal atoms can be anchored and maintained in high dispersion [52]. For instance, isolated Pt atoms have been firmly trapped by the four pyrrolic nitrogen sites of porphyrin centers in MOFs through $\mathrm{Pt}(\mathrm{II})$ metalation and a followed reduction process [53].

Besides, the MOFs are widely used as the precursors for preparing SACs with a particular configuration. When bimetallic $\mathrm{Zn} / \mathrm{Co} \mathrm{MOF}$ is selected as a precursor for calcination, isolated $\mathrm{Co}$ atoms decorated porous carbon matrix can be constructed with the high loading content over $4 \mathrm{wt} \%$ [54]. In this process, Co nodes can be in situ-converted into isolated Co atoms by carbonization of the organic linker. The intentional mixed $\mathrm{Zn}$ atoms are volatile and can be easily removed at high temperatures, which can serve as a "fence" to avoid the generation of $\mathrm{Co}-\mathrm{Co}$ bonds. The final single Co catalyst exhibits pronounced oxygen reduction reaction (ORR) activity with a more positive half-wave potential $(0.881 \mathrm{~V})$ than that of commercial Pt/C (Fig. 2d). Taking advantage of the uniformly distributed pores in MOFs, isolated metal atoms can be confined in the MOFs matrix [55]. $\mathrm{Li}$ et al. have reported the host-guest strategy to adsorb $\mathrm{FeCl}_{3}$ molecules within the confined space of $\mathrm{Zn} / \mathrm{Co}$ bimetallic MOFs [56]. Fe ions can be confined in the graphitization process and coordinated with adjacent Co atoms, achieving an elegant hybrid with porphyrin-like Fe-Co dual sites embedded carbon matrix.

\subsection{Defect Immobilized Approaches}

Defects exist widely in nanomaterials, such as step edges, caves and intrinsic defects, which are beneficial to immobilize isolated metal atoms and alter the geometric and electronic properties for improving the reaction activity [57, 58].

Step edges are the most ubiquitous defects on the solid surfaces. Edges in the ceria have been applied for segregating the $\mathrm{Pt}$ atoms in the planar $\mathrm{PtO}_{4}$-configuration [59]. Experiments and density functional theory (DFT) results confirm the preferential segregation of Pt atoms at the steps with direct Pt-O bonding. The density of the step edges can be well tuned and thus afford the SACs with varied Pt loading amounts. Yan and coworkers have taken advantage of the fourfold caves in the phosphomolybdic acid for stabilizing the isolated Pt sites in high loading content [60]. The isolated Pt reactive centers are coordinated by four oxygen atoms in a distorted square-planar geometry. The developed catalyst exhibits impressive activity in hydrogenation of nitrobenzene and cyclohexanone. The intrinsic defects not only can be applied as the anchor sites, but also perform as the media for modulating the intrinsic catalytic activity of SACs. Li et al. have decorated the isolated Pt atoms on the intrinsic defects of the $\mathrm{TiO}_{2}$ support $\left(\mathrm{Pt}_{1} / \mathrm{def}^{-\mathrm{TiO}_{2}}\right)$ as a superb photocatalyst (Fig. 2e-h) [61]. Notably, isolated Pt atoms can promote the adjacent $\mathrm{TiO}_{2}$ moieties to produce surface oxygen vacancies and $\mathrm{Ti}^{3+}$ defects, giving rise to an exceptional photocatalytic performance for hydrogen evolution.

\subsection{Other Synthesis Methods}

In the synthesis progress of SACs, some other approaches have also been well established. In this section, a brief introduction of other synthesis methods is provided, including atomic layer deposition (ALD), ball-milling, and 
high-temperature atom trapping. It should be noted that these methods typically involve expensive equipment and afford low yields, which are not favorable for large-scale production.

ALD is one of the high-vacuum physical deposition techniques, which involves the self-limiting binary reactions between the substrate and gaseous precursor and allows precisely controlled deposition of diverse structures on various substrates. In the ALD process, the chosen substrate is alternately exposed to various vapor precursors. The metal is deposited in an atomic layer-by-layer manner. Sun et al. have stabilized the isolated Pt atoms on the surface of graphene nanosheet via ALD technique [62]. When the support is exposed to (methylcyclopentadienyl)-trimethylplatinum $\left(\mathrm{MeCpPtMe}_{3}\right)$, the limited supply of oxygen on the graphene nanosheets disenables the oxidization of full ligands, generating Pt species in monolayer. Subsequently, the new oxygen layer can be formed on the $\mathrm{Pt}$ with further exposure to the oxygen, which can continue to adsorb Pt in a layer-by-layer manner. The deposition of Pt can be precisely controlled by adjusting the cycle numbers of ALD. These single Pt atom catalysts exhibit much higher activity in the methanol oxidation reaction and $\mathrm{CO}$ adsorption. However, this route is limited by the high cost and rigorous manipulation. The catalysts obtained via ALD approach usually possess nonuniform size and shape, which is not favorable for deeply understanding the correlation between local structures and reaction activities.

High-energy ball milling approach enables the breaking and reconstruction of chemical bonds in molecules and thus shows great potential to synthesize SACs. Bao and colleagues have reported the preparation of graphene confined metal- $\mathrm{N}_{\mathrm{x}}$ catalysts via the ball milling approach for the first time [63-65]. In the synthesis process, graphene nanosheets (GNs) are firstly obtained by ball milling the natural graphite powder, then iron phthalocyanine $(\mathrm{FePc})$ is combined with the GNs for further ball milling. Due to the high energy input by the ball milling, FePc can be reconstructed, affording the $\mathrm{FeN}_{4}$ embedded graphene.

The SACs can also be constructed through the thermal transformation of bulk particles into isolated reactive centers. Datye et al. have employed ceria powders with different exposed surface facets as supports to trap the individual platinum atoms [66]. When Pt particles deposited $\mathrm{Al}_{2} \mathrm{O}_{3}$ $\left(\mathrm{Pt} / \mathrm{La}-\mathrm{Al}_{2} \mathrm{O}_{3}\right)$ mixing with ceria powders, $\mathrm{Pt}$ species can be emitted under high-temperature treatment and then migrate to the surface of $\mathrm{CeO}_{2}$ spontaneously. Various characterizations show that ceria polyhedrons and nanorods behave more effectively in avoiding the aggregation of $\mathrm{Pt}$ when comparing with the ceria cubes. $\mathrm{Li}$ and coworkers have further developed the thermal transformation strategy for converting the nanoparticles of precious metal $(\mathrm{M}=\mathrm{Pt}, \mathrm{Pd}$, and $\mathrm{Au})$ to isolated reactive centers in the inert atmosphere [67]. The mobile precious metal atoms are stabilized by the defects of nitrogen-doped carbon in the $\mathrm{M}-\mathrm{N}_{4}$ geometry, providing driven force for the conversion of the nanoparticle to the isolated atoms.

\section{Coordination Engineering to Explore the Intrinsic Activity}

Evaluating the contribution of the particular active site is an ideal approach to understand the origin of the catalytic process. SACs with the well-controlled configuration and homogeneous dispersion provide us an elegant platform for identifying the contribution of the isolated active sites and recognizing the key parameters for influencing the catalytic performance $[60,68,69]$. Thus, modulating the coordination spheres of SACs is an effective strategy to further explore the intrinsic activity of the catalysts.

\subsection{Tuning the Coordination Species of Active Centers}

It is noteworthy that the active centers with nearly identical coordination sphere, but varied coordination species deliver distinct catalytic activities. The coordination species is a quite crucial parameter for influencing the catalytic performance. Patzke and colleagues have confirmed the important role of metal species in tuning the electrocatalytic performance for ORR (Fig. 3a) [70]. They have developed a softlanding molecular approach to decorate metal phthalocyanines with varied metal species $(\mathrm{M}=\mathrm{Co}, \mathrm{Ni}$, and $\mathrm{Fe})$ onto the graphene oxide layers, and successfully synthesized the well-defined SACs. Experimental results and DFT calculations suggest that the $\mathrm{Fe}$ species exhibits stronger affinity toward $\mathrm{O}_{2}$ species when comparing with other metal species, resulting in greatly accelerated reaction kinetics for the oxygen reduction.

Besides, introducing the exotic non-metal species into the coordination sphere is also an effective approach for 
(a) NiPc-GO CoPc-GO FePc-GO
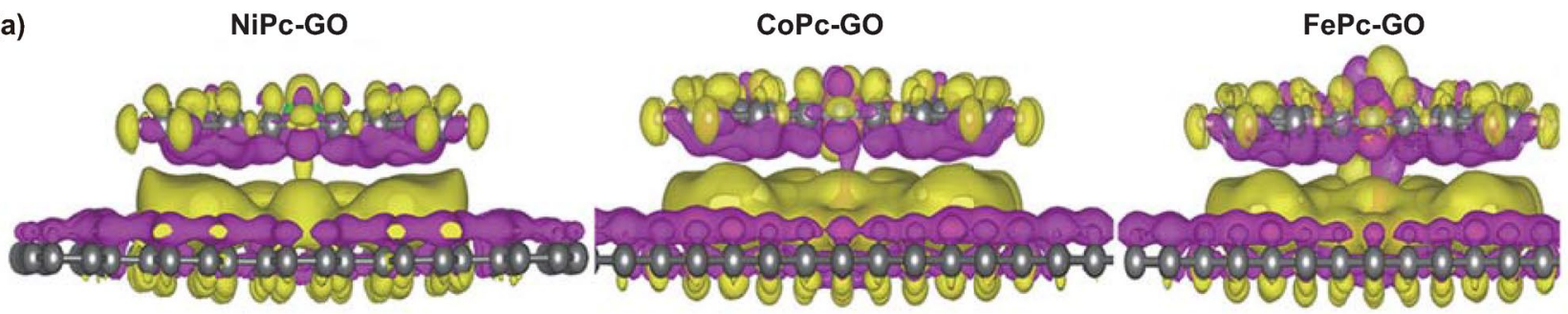

(b)

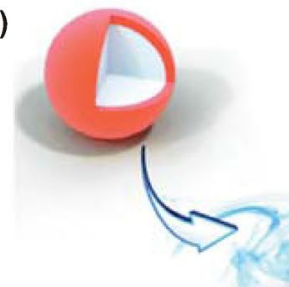

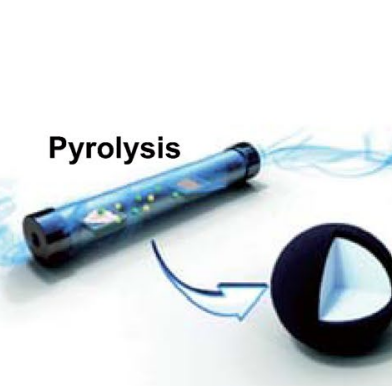

(d)

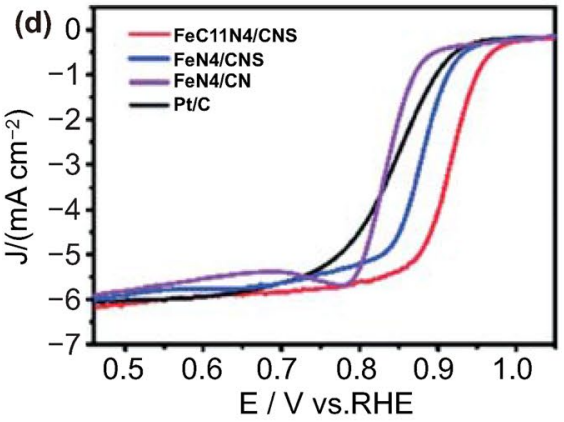

(g)

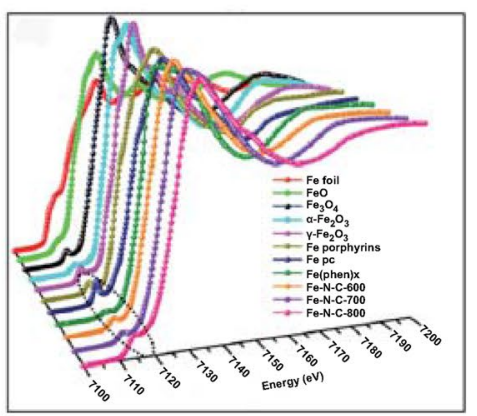

(e) HAADF-STEM

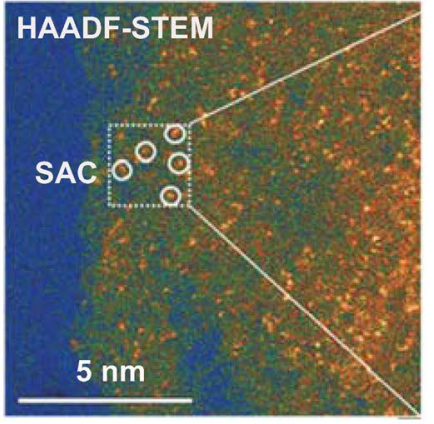

(h)

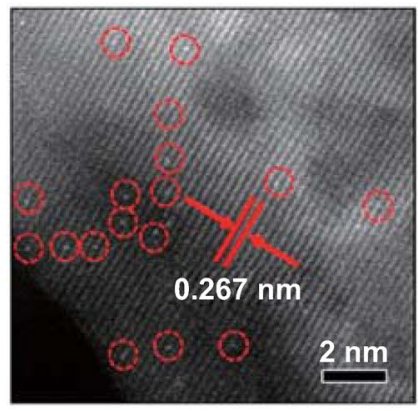

(c)

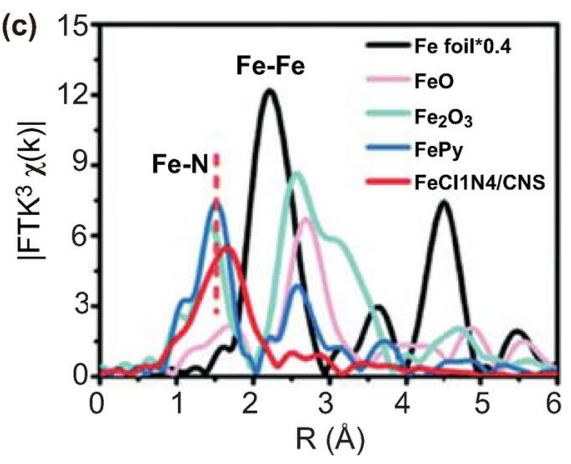

(f)

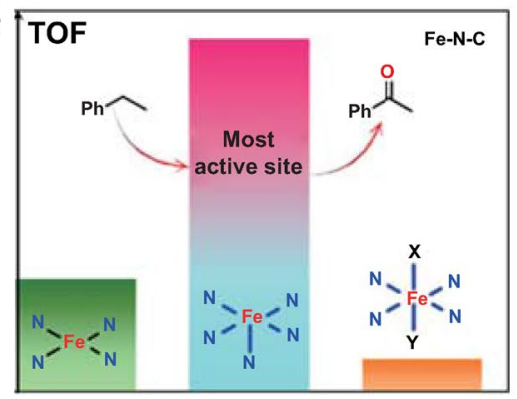

(i)

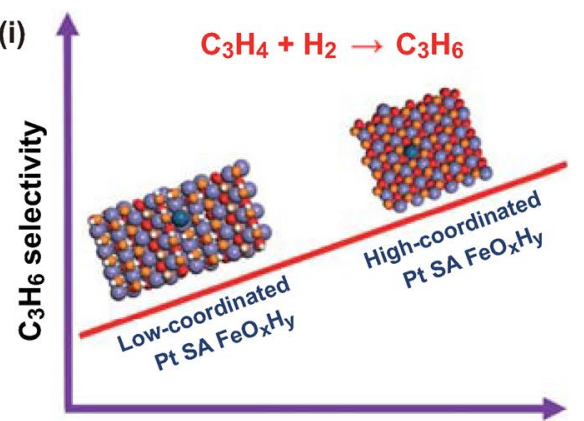

Pt-O coordination number

Fig. 3 a DFT simulations of the synergistic effect between MPcs $(\mathrm{M}=\mathrm{Ni}, \mathrm{Co}, \mathrm{Fe})$ and GO. Isosurface plots of charge redistribution for NiPcGO, CoPc-GO and FePc-GO. Yellow or violet color represents the accumulation or depletion of electrons, respectively. Reproduced from Ref. [70] with permission. Copyright 2020 American Chemical Society. b Schematic illustration of the synthesis of $\mathrm{FeCl}_{1} \mathrm{~N}_{4} / \mathrm{CNS}$ c Fourier transform (FT) at the Fe K-edge of the $\mathrm{FeCl}_{1} \mathrm{~N}_{4} / \mathrm{CNS}$, FePy, $\mathrm{FeO}, \mathrm{Fe}_{2} \mathrm{O}_{3}$ sample and $\mathrm{Fe}$ foil. d ORR polarization curves in $\mathrm{O}_{2}$-saturated $0.1 \mathrm{M}$ KOH. b-d Reproduced from Ref. [72] with permission. Copyright 2018 The Royal Society of Chemistry. e HAADF-STEM images of Fe-N-C. f $\mathrm{Fe}^{\mathrm{III}} \mathrm{N}_{5}$ affords the highest turnover frequency for the selective oxidation of the $\mathrm{C}-\mathrm{H}$ bond. $\mathrm{g}$ The normalized XANES spectra at the Fe K-edge of different samples. The dotted ellipse shows the pre-edge peak at $7117 \mathrm{eV}$. e-g Reproduced from Ref. [73] with permission. Copyright 2017 American Chemical Society. h High-resolution AC-STEM image of isolated Pt atoms. $\mathbf{i}$ The propylene selectivity increases as the coordination number of Pt-O increases. h-i Reproduced from Ref. [76] with permission. Copyright 2020 American Chemical Society 
(a)
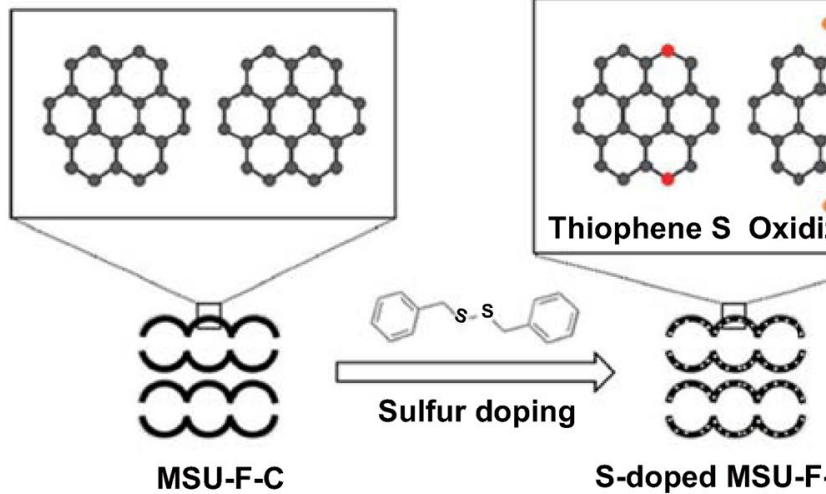

S-doped MSU-F-C

(S-MSUFC)
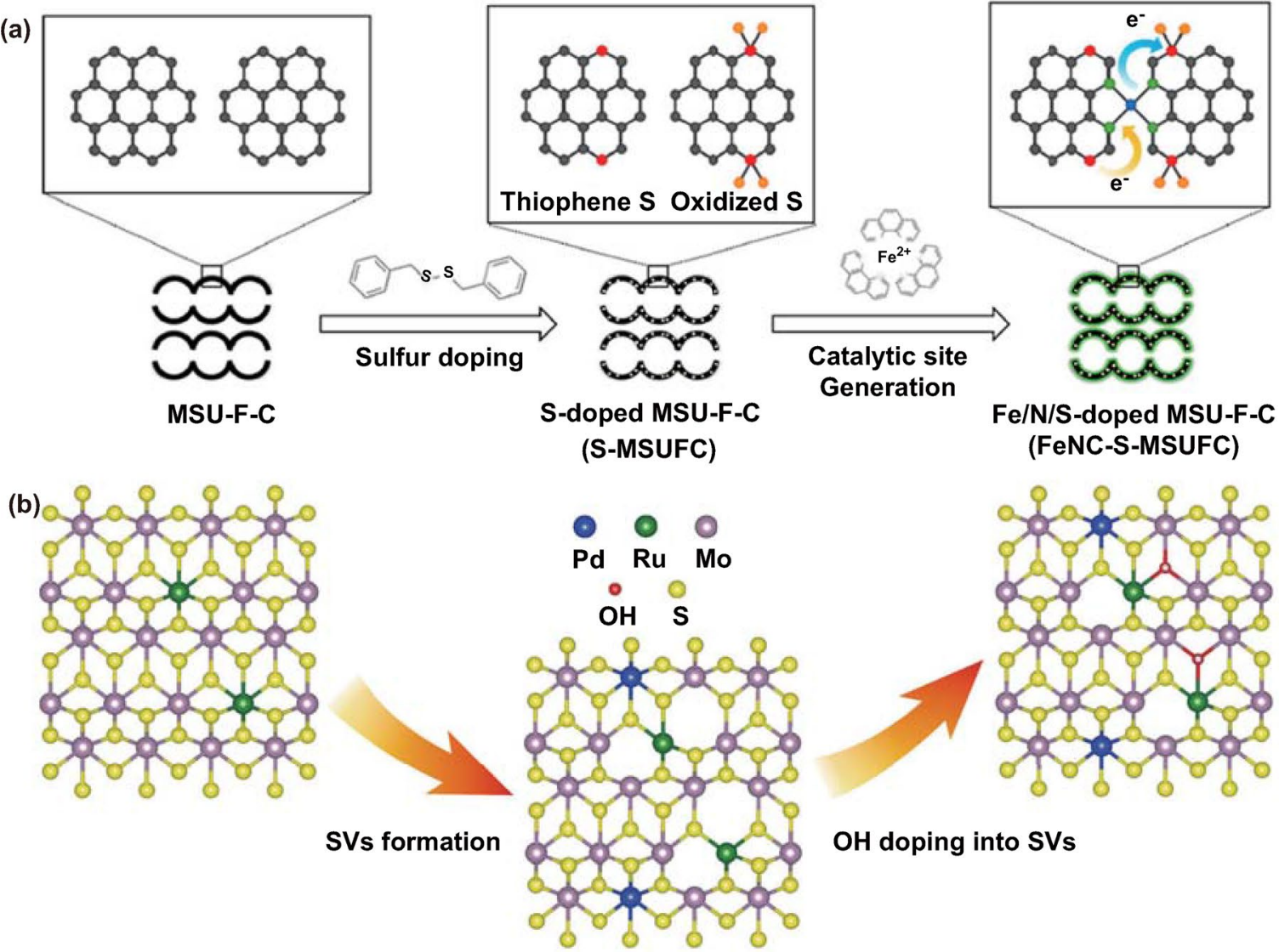

SVs formation

(c)

(d) $F$
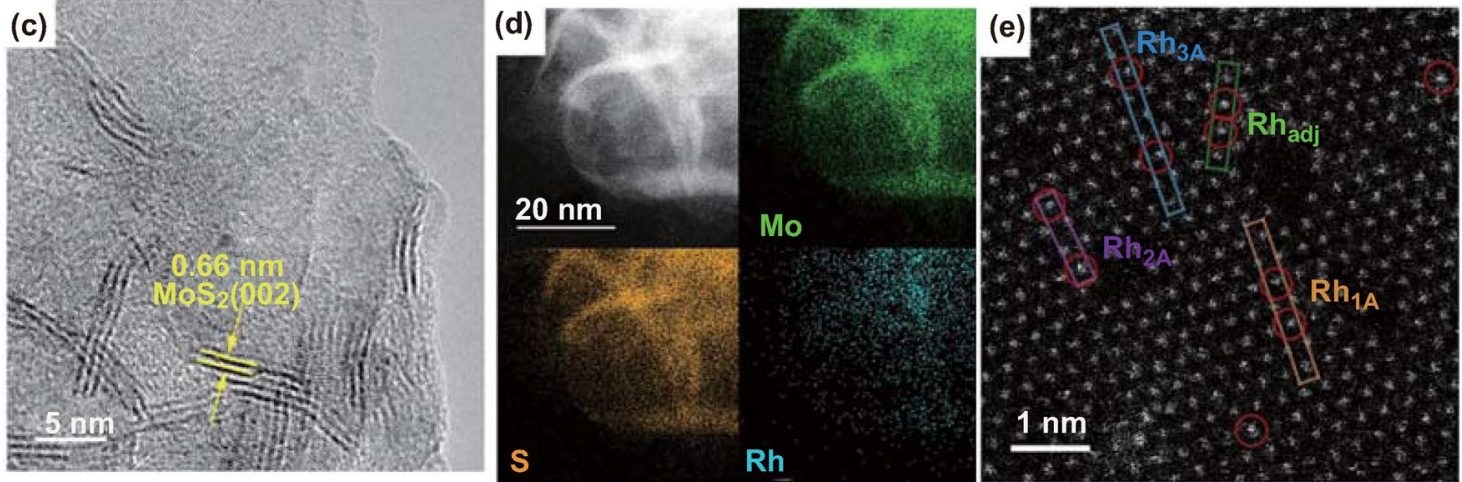

(f)
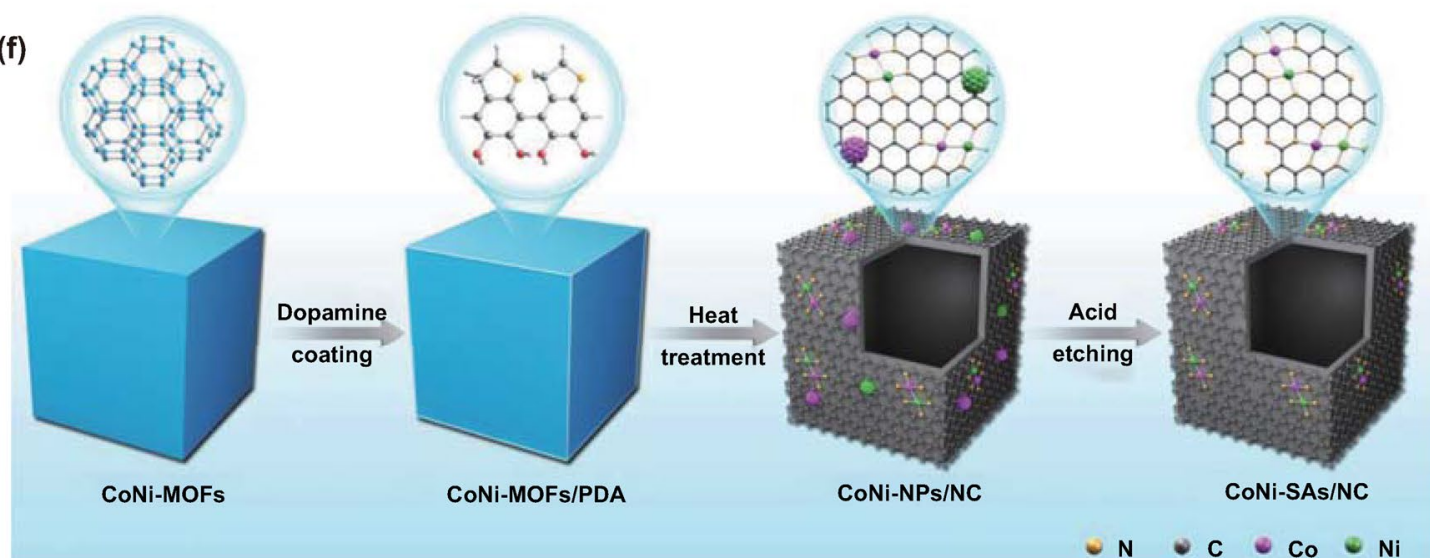
\Fig. 4 a Schematic representation of the synthesis of $\mathrm{Fe} / \mathrm{N} / \mathrm{C}$ catalysts with S-doped MSU-F-C. In the figure, the black, light green, blue, red, and orange spheres represent carbon, nitrogen, iron, sulfur, and oxygen atoms, respectively. Reproduced from Ref. [78] with permission. Copyright 2019 American Chemical Society. b Schematics for the synthesis strategy of Pd, Ru dual-doped $\mathrm{MoS}_{2-x} \mathrm{OH}_{\mathrm{y}}$ phase. Blue, yellow, purple, green and red spheres represent Pd, S, $\mathrm{Mo}, \mathrm{Ru}$, and $\mathrm{O}$ atoms, respectively. Reproduced from Ref. [80] with permission. Copyright 2020 Springer Nature. c HRTEM image and d HAADF-STEM image with EDS mapping of the Rh-MoS ${ }_{2}$ sample. e Atomic-resolution HAADF-STEM image of the $\mathrm{Rh}-\mathrm{MoS}_{2}$ sample. $\mathrm{Rh}_{\text {adj }}$ denotes the distribution of confined $\mathrm{Rh}$ atoms adjacent to each other. $\mathrm{Rh}_{\mathrm{nA}}$ denotes the distribution of confined $\mathrm{Rh}$ atoms separated by $\mathrm{n}$ Mo atoms along the armchair direction. c-e Reproduced from Ref. [84] with permission. Copyright 2020 Wiley-VCH Verlag $\mathrm{GmbH} \& \mathrm{Co}$. KGaA, Weinheim. f Schematic illustration of the synthetic strategy of CoNi-SAs/NC. Reproduced from Ref. [87] with permission. Copyright 2019 Wiley-VCH Verlag GmbH \& Co. KGaA, Weinheim

adjusting the electronic structure of the reactive center. Zhang and co-workers have successfully introduced the $\mathrm{N}$ species into the first coordination sphere of the isolated $\mathrm{Ni}$ atoms [71]. Comparing with the Ni-C dominated coordination sphere, the $\mathrm{Ni}-\mathrm{N}$ coordination with effective electron coupling can move down the Fermi level of the hybrid, resulting in greatly reduced adsorption energy of intermediates and highly accelerated reaction kinetics for oxygen evolution.

Li and colleagues have further introduced chlorine atoms into the coordination sphere of the isolated Fe centers, and constructed an elegant catalyst $\left(\mathrm{FeCl}_{1} \mathrm{~N}_{4} / \mathrm{CNS}\right)$ for electrochemical ORR (Fig. 3b-d) [72]. Comparing with the catalyst $\mathrm{FeN}_{4} / \mathrm{CNS}$ without chlorine modification, the catalyst $\mathrm{FeCl}_{1} \mathrm{~N}_{4} / \mathrm{CNS}$ delivers greatly improved catalytic activity with a half-wave potential of $0.921 \mathrm{~V}$. Calculation results reveal that the exceptionally outstanding ORR activity can be ascribed to the electronic coupling between the Fe reactive centers and coordinated chlorine atoms, resulting in heavily modified electronic structures and highly accelerated reaction kinetics.

\subsection{Controlling the Coordination Number of the Active Center}

Given that the isolated reactive centers in SACs are stabilized by direct ionic and covalent coordination with the supports, the coordination number of the active sites should be an effective and reasonable descriptor for modulating the intrinsic activity of the SACs. Zhang and coworkers have successfully fabricated the exclusively dispersed Fe catalysts with different coordination numbers $\left(\mathrm{FeN}_{\mathrm{x}}, \mathrm{x}=4-6\right)$ (Fig. 3e-g) [73]. Combinatorial investigations demonstrate that $\mathrm{Fe}^{\mathrm{III}} \mathrm{N}_{5}$ delivers the highest catalytic performance for selective oxidation of $\mathrm{C}-\mathrm{H}$ bond, which is one order magnitude and three times higher than those of $\mathrm{Fe}^{\mathrm{III}} \mathrm{N}_{6}$ and $\mathrm{Fe}^{\mathrm{II}} \mathrm{N}_{4}$, respectively. The low activity of $\mathrm{Fe}^{\mathrm{III}} \mathrm{N}_{6}$ should be ascribed to its saturated coordination between central Fe and surrounding $\mathrm{N}$ atoms, and no coordination space is left for reacting with the reactant. Zhao and colleagues have further decorated the isolated tungsten atoms onto the nitrogen-doped carbon nanosheets with precisely controlled W-N coordination numbers [74]. Experimental results clearly demonstrate that the isolated $\mathrm{W}$ atom coordinated by five nitrogen atoms exhibits remarkably enhanced ORR catalytic performance relative to those hybrids with $\mathrm{W}-\mathrm{N}$ coordination number of 3 and 4 . The coordination number-sensitive ORR performance of the single tungsten atom catalyst should be originated from the interaction between $\mathrm{OH}^{-}$and the isolated $\mathrm{W}$ atoms moderated by the coordination number.

Coordination chemistry of the isolated reactive centers supported on metal oxide has also been explored with obvious progress. Parkinson et al. have decorated isolated Ir atoms into the lattice of $\mathrm{Fe}_{3} \mathrm{O}_{4}$ with varied geometries and applied the as-synthesized hybrids for $\mathrm{CO}$ adsorption [75]. Both the fivefold Ir atoms with octahedral coordination sphere and twofold Ir atoms with square-planar configuration exhibit greatly enhanced $\mathrm{CO}$ adsorption ability when comparing with the metallic Ir. Experimental results and DFT simulation clearly demonstrate the coordination unsaturated Ir atoms with coordination vacancies can effectively reactive with the $\mathrm{CO}$ molecules and thus boost the adsorption capacity.

Zhang and colleagues have further demonstrated that varying the coordination number of the isolated Pt centers can obviously improve the propylene selectivity during propyne semi-hydrogenation (Fig. 3h, i) [76]. They have modulated the coordination number of the isolated Pt centers from 3.43 to 5.04 over $\mathrm{FeOOH}$ supports by the thermal treatment. Experimental results and DFT simulations verify that the $\mathrm{Pt} \mathrm{SACs}$ with reduced $\mathrm{Pt}-\mathrm{O}$ coordination 
number is beneficial for propyne over-hydrogenation, resulting in greatly enhanced catalytic activity.

\subsection{Heteroatoms Interactions within the Support}

Heteroatoms doping within the support has been recently developed as a simple and effective strategy to modulate the electronic structure of the isolated reactive centers. Guo and co-workers have synthesized a sulfur-doped electrocatalyst (Fe/SNC) with high density of atomically dispersed Fe sites [77]. The incorporated sulfur gives rise to a thiophene-like structure (C-S-C) in Fe/SNC. The localized electron density around $\mathrm{Fe}$ atoms can be reduced by the C-S-C moieties, leading to improved interaction with oxygenated species and boosted ORR activity. Lee and coworkers have decorated electron-withdrawing oxidized $\mathrm{S}$ group into the carbon plane and applied the as-synthesized hybrids for ORR (Fig. 4a) [78] . The electron-withdrawing effect can lower the d-orbital energy level of the isolated $\mathrm{Fe}$ atoms and thus boost the ORR activities. In contrast, the thiophene-like $\mathrm{S}$ functionalities behaving as electron donator to the carbon plane generate stronger adsorption of the ORR intermediates on the $\mathrm{Fe}-\mathrm{N}_{4}$ sites and reduce the specific activity of ORR.

$\mathrm{Li}$ and co-workers have further modulated the electronic states of the active centers via decorating the phosphorus and sulfur species into the carbon matrix [79]. Experimental results and theory simulation suggest that the effective electron coupling between the isolated $\mathrm{Fe}$ atoms and surrounding $\mathrm{S}$ and $\mathrm{P}$ atoms should be responsible for the high-efficiency and satisfactory kinetics of ORR. Ge et al. have built a di-anionic surface on metal doped $\mathrm{MoS}_{2}$, in which sulfur anions can be molecularly substituted by $-\mathrm{OH}$ (Fig. 4b) [80]. The -OH group endows the interface with reactant dragging functionality. The well-conditioned surface, in conjunction with activated sulfur atoms by heteroatom metal doping as active sites, results in the greatly boosted hydrogen evolution reaction (HER) kinetics. The compositional advantage is also proved by introducing other metal species. The bimetallic catalyst of isolated $\mathrm{Zn}$ and $\mathrm{Co}$ atoms confined in sulfur-modified carbon architecture ( $\mathrm{ZnCo} / \mathrm{NSC}$ ) shows an impressive ORR performance with the half-wave potential of $0.893 \mathrm{~V}$, which outperforms the catalysts with only Zn or Co metallic sites [81]. Investigations demonstrate that interaction between the bimetallic sites can activate the $\mathrm{O}-\mathrm{O}$ bonding and reduce the reaction barriers in the ORR process.

\subsection{Synergetic Interaction between Neighboring Metal Monomers}

As the density of isolated active centers increases, the distance between two metal monomers can be greatly shortened and neighboring metal monomers are formed. The interaction between a pair of active monomers can generate a more optimized electronic structure and thus influence the related catalytic performance. Zeng and coworkers have demonstrated that pairs of Pt monomer stabilized by $\mathrm{MoS}_{2}$ can work in synergy to enhance the $\mathrm{CO}_{2}$ hydrogenation catalytic performance and undergo different reaction paths relative to isolated $\mathrm{Pt}$ monomer [82]. Formic acid and methanol are obtained in the hydrogenation of $\mathrm{CO}_{2}$ catalyzed by neighboring Pt monomers. In the absence of ensembles, isolated $\mathrm{Pt}$ atoms catalyze $\mathrm{CO}_{2}$ into methanol instead of the formic acid intermediates. Li et al. have synthesized the diatomic $\mathrm{Fe}_{2}$ clusters supported on $\mathrm{g}-\mathrm{C}_{3} \mathrm{~N}_{4}$, which exhibit outstanding catalytic performance for the alkene epoxidation [83]. The unique reactivity is benefited from the generated active oxygen species from the diatomic $\mathrm{Fe}_{2}$ clusters. In contrast, the isolated $\mathrm{Fe}$ atom catalyst is nearly inert. Deng et al. have realized an impressive HER activity by adjusting the distance between the isolated $\mathrm{Rh}$ atoms on $\mathrm{MoS}_{2}$ (Fig. 4c-e) [84]. DFT simulation further suggests that the optimized distance can trigger the activity of neighboring $\mathrm{S}$ atoms for hydrogen evolution, thus resulting in the maximized activity on the activated $\mathrm{S}$ atoms in the hybrid.

Employing two different neighboring monomers can provide a new reaction path to boost the catalytic selectivity and activity. Guo and coworkers have reported the neighboring $\mathrm{Pt}-\mathrm{Ru}$ monomers stabilized by the $\mathrm{N}$ vacancy on $\mathrm{g}-\mathrm{C}_{3} \mathrm{~N}_{4}$ [85]. The neighboring Pt-Ru monomers with rich electrons are expected to own a higher activity than the $\mathrm{Ru}-\mathrm{Ru} / \mathrm{Pt}-\mathrm{Pt}$ monomers or single $\mathrm{Ru} / \mathrm{Pt}$ atom in the $\mathrm{CO}$ oxidation reaction. Mechanism investigations suggest that $\mathrm{Pt}-\mathrm{Ru}$ pairs enable the formation of bridge-type $\mathrm{O}_{2}$ adsorption and thus optimize the $\mathrm{O}_{2}$ activation process. Sun and coworkers have fabricated discrete $\mathrm{Zn}$-Co dual atomic centers on nitrogendoped carbon to offer more concentrated active sites ( $\mathrm{Zn} /$ CoN-C) [86]. Structural information and DFT calculations reveal that electronic structure can be modulated due to the formation of $\mathrm{ZnCoN}_{6}$ site, elongating the $\mathrm{O}-\mathrm{O}$ bond length and accelerating the breaking of $\mathrm{O}_{2}$. The as-synthesized $\mathrm{Zn} /$ CoN-C catalyst delivers impressive ORR activities in both 
acid and alkaline media with a half-wave potential of 0.796 and $0.861 \mathrm{~V}$, respectively. Hu et al. have synthesized binary Co-Ni sites anchored by N-doped hollow carbon nanocubes (CoNi-SAs/NC) (Fig. 4f) [87]. The synergistic effect of diatomic $\mathrm{Co}-\mathrm{Ni}$ sites in porous carbon matrix endows CoNi-SAs/NC with optimized oxygen adsorption/desorption properties and decreases the reaction barriers for the oxygen involved catalysis, accelerating the bifunctional oxygen reduction/evolution reaction kinetics, surpassing other counterpart catalysts.

\subsection{Spatial Microenvironment}

Spatial confinement has been established as an effective strategy to develop SACs with impressive activity, selectivity and stability. Han and colleagues have realized the confinement of the isolated Mo atoms in the porous ZSM-5 matrix [88]. The integrated differential phase-contrast scanning transmission electron microscopy directly confirms the atomic dispersion of Mo species in the pores of the ZSM-5 support (Fig. 5a-d). Moreover, the specific Mo-Al interaction provides an opportunity for relocating the $\mathrm{Al}$ atoms in the framework of the ZSM-5. Lou and coworkers have developed an effective strategy for confining the isolated $\mathrm{Pt}$ atoms into the porous carbon matrix, which exhibits greatly boosted mass activity for electrocatalytic HER comparing with commercial Pt/C catalyst (Fig. 5e, f) [89]. Qiu and coworkers have decorated the isolated Pd atoms into the pores of MIL-101 $(\mathrm{Cr})$, which delivers much higher hydrogenation activity than that of Pd adsorbed on the MIL-101 surface [90]. The authors ascribe the superior catalytic activity to the highly exposed Pd atom and confinement effect offered by the pores of MOFs.

Decorating the isolated metal species into the regular pores of the support can integrate their respective merits for catalysis. Hyeon et al. have confirmed the important role of the porous $\mathrm{N}$-doped carbon structure of $\mathrm{Fe}-\mathrm{N}-\mathrm{C}$ catalysts for electrochemical ORR activity [91]. With the existence of mesopores, a large part of the physical surface area becomes reachable for the reactants and thus facilitates the kinetic accessibility of the active sites during the ORR. Moreover, porous zeolites and MOFs can serve as novel molecular sieves. Yu and colleagues have employed the oxygen atoms of MFI-type zeolites to stabilize isolated Ru atoms [92]. The zeolite channels facilitate shape selectivity in a manner that allows nitrobenzene with a smaller size to reach $\mathrm{Ru}$ sites while the bulkier 3-nitrotoluene is hindered by the zeolite pores. Similarly, the ZIF-8 pore structure allows contact of the reactant with the anchored Ru sites with absolute regionselectivity of catalyzing terminal alkynes but not internal alkynes (Fig. 5g, h) [93].

Defects are commonly existed in the spatial microenvironment and may cast some positive effects on the catalytic performances. Controlled construction of surface defects on supports has also been proven effective for developing SACs with well-defined microenvironments. The isolated reactive centers locating at the corner and edge sites always display a superior activity compared with the reactive centers confined in the bulk phase. Inspired by this, researchers set out to utilize highly curved supports to trap single metal sites. For example, edge-hosted $\mathrm{Fe}-\mathrm{N}_{4}$ moieties have been prepared in hierarchically micro-mesoporous carbon matrix (Fig. 5i), which exhibit greatly improved ORR activity compared with those of intact atomic configuration [94]. Both experiments and DFT calculations reveal that such a defective $\mathrm{Fe}-\mathrm{N}_{4}$ single site is a key to tailor the bonding structures of $\mathrm{N}$ and lower the overall ORR barriers. Song et al. have used onion-like carbon, instead of two-dimensional graphene, to support single $\mathrm{Pt}$ atoms and achieved the outstanding HER performance [95]. The working mechanism is uncovered by DFT results. Compared to the conventional catalysts, onion-like carbon with curve nature enables the accumulation of electrons around the Pt regions. The strong local electric field accelerates charge delivery and optimizes catalytic kinetics for HER. Lou et al. have implanted single $\mathrm{Ru}$ atoms with precise configuration into edge-rich carbon (ECM@Ru) for the electrocatalytic hydrogen evolution [96]. Comparing with the Ru-decorated carbon matrix (CM@Ru) particles without edges, ECM@ Ru displays a 4.1 times higher mass activity at the overpotential of $100 \mathrm{mV}$. Experimental and calculation results reveal the key role of carbon edges in enhancing the local electric field around $\mathrm{Ru}$ monomers and accelerating the HER kinetics. They have also dispersed $\mathrm{Ru}$ atoms on multi-edged $\mathrm{TiO}_{2}$ spheres for photocatalytic hydrogen evolution [97]. The multiedge $\mathrm{TiO}_{2}$ architecture greatly facilitates the charge separation and transport in photocatalysis process, further demonstrating the important role of the defects and edges in accelerating the reaction kinetics. 

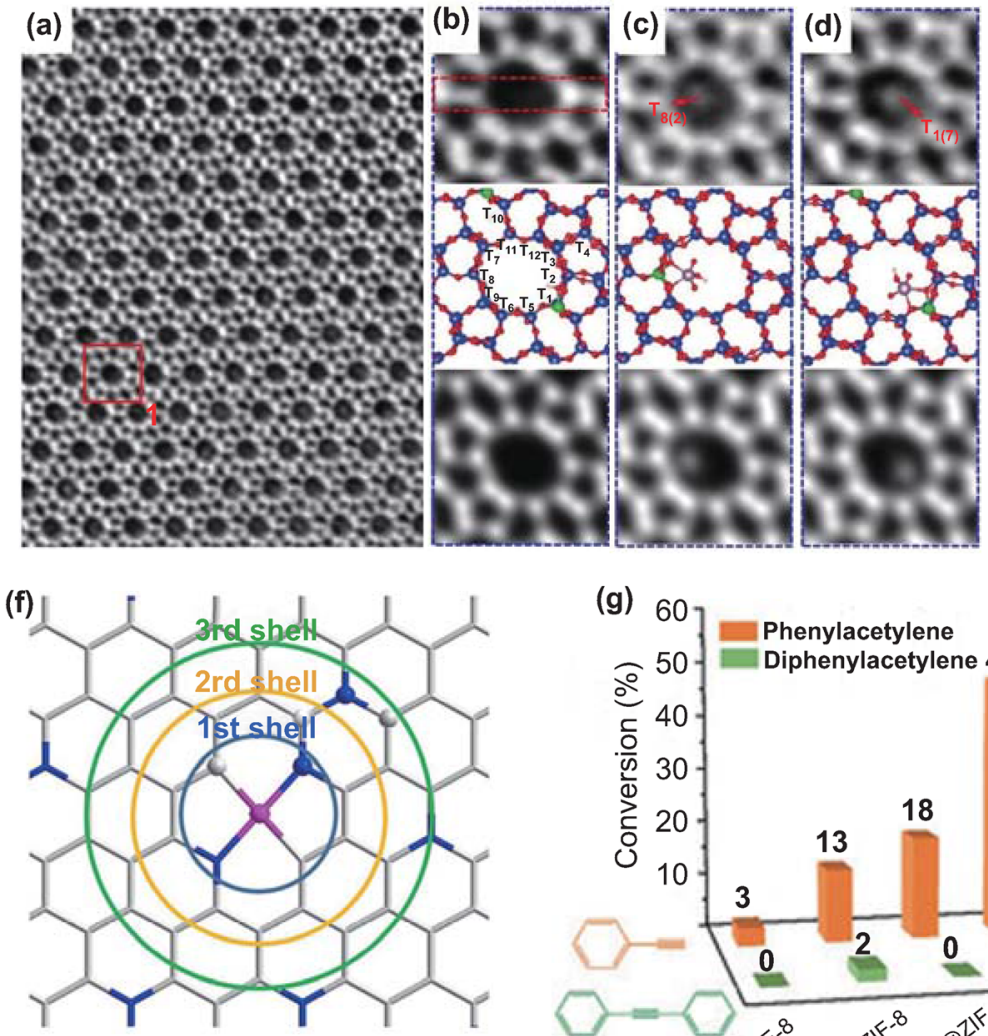
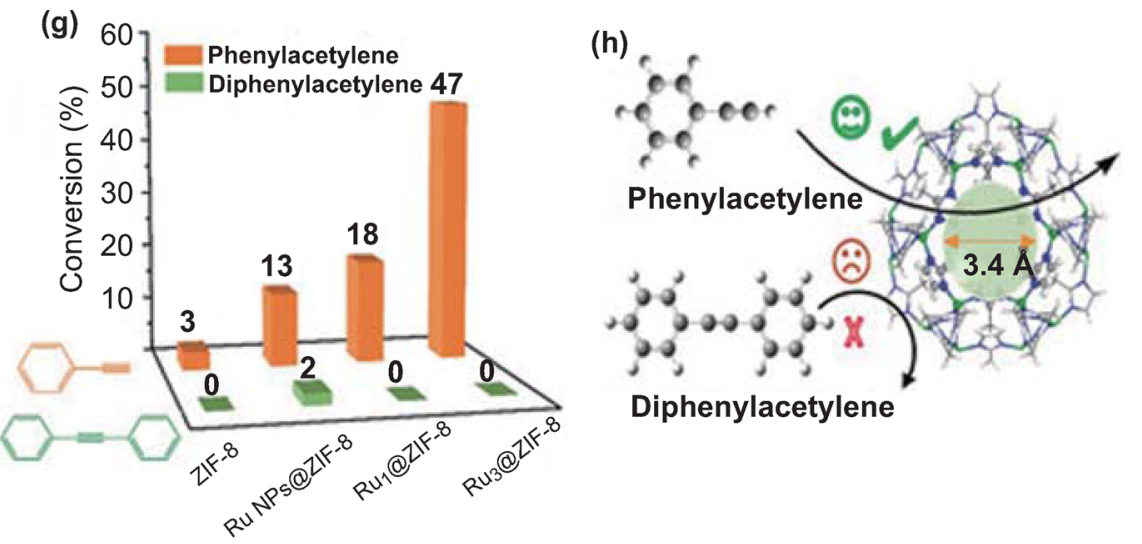

Diphenylacetylene

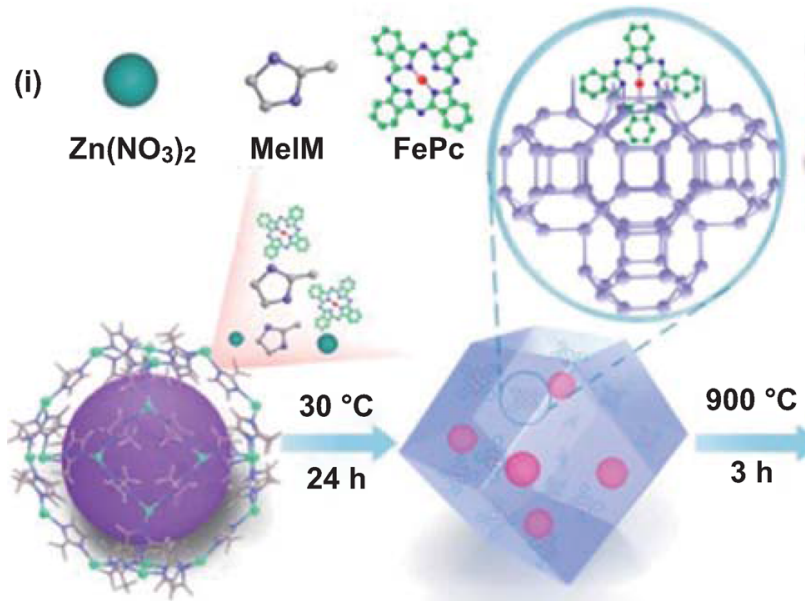

FePc-X@ZIF-8

Multimolecular Aggregates

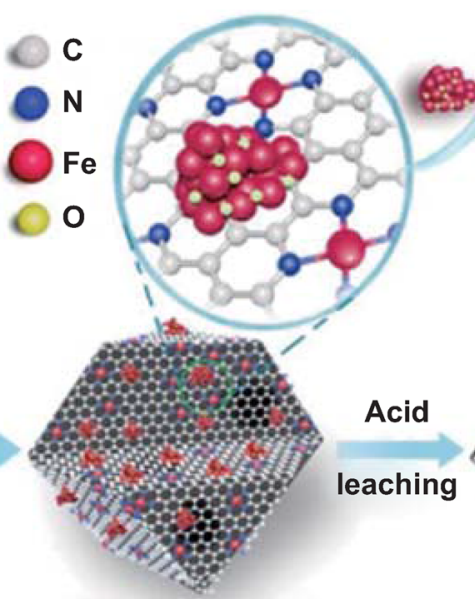

$\mathrm{Fe} \mathrm{SAs} \mathrm{\&} \mathrm{Fe}_{2} \mathrm{O}_{3}-\mathrm{N} / \mathrm{C}-\mathrm{x}$ $\mathrm{Fe}_{2} \mathrm{O}_{3}$

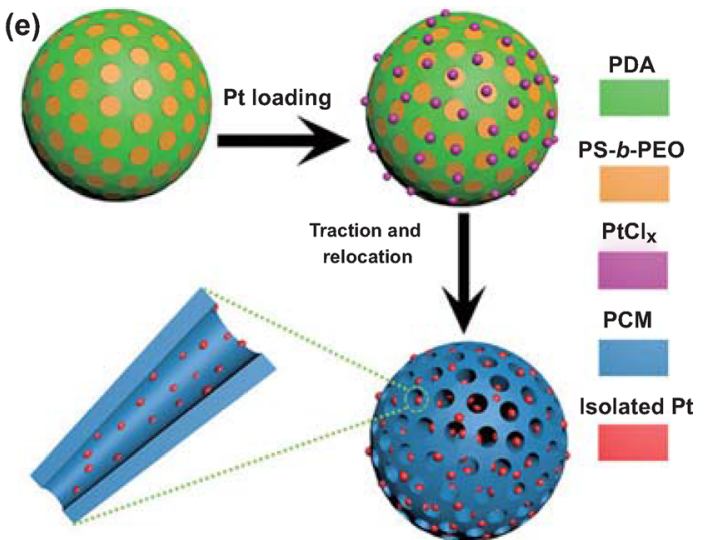

Fig. 5 a A representative iDPC-STEM image of Mo/ZSM-5, showing the presence of off-center contrast in many 10-MR channels. b-d Zoomed-in areas of a, corresponding to three scenarios: empty channel $\mathbf{b}$, and channels containing a $\mathrm{MoO}_{3} \mathrm{H}$ cluster bound at the T8 site $\mathbf{c}$ and at the T1 site d. Each panel includes the raw image (top), the calculated structural model (middle), and the simulated projected electrostatic potential (bottom). Si blue, O red, Al green, Mo pink, H white. a-d Reproduced from Ref. [88] with permission. Copyright 2020 Wiley-VCH Verlag GmbH \& Co. KGaA, Weinheim. e Schematic illustration of the synthetic procedure of Pt@PCM. f Schematic description for the coordination shells for the isolated Pt over the graphene. e-f Reproduced from Ref. [89] with permission. Copyright 2018, American Association for the Advancement of Science. $\mathbf{g}$ Conversion for hydrogenation of phenylacetylene over $\mathrm{Ru}_{3} @ \mathrm{ZIF}-8, \mathrm{Ru} 1 \mathrm{ZIF-8}$ and references. $\mathbf{h}$ Molecular sieving size-selectivity sketch of phenylacetylene and diphenylacetylene. g-h Reproduced from Ref. [93] with permission. Copyright 2019 Wiley-VCH Verlag GmbH \& Co. KGaA, Weinheim. i Schematic illustration of $\mathrm{Fe}_{-} \mathrm{N}_{4}$ sites anchored on 3D hierarchically porous carbon. Reproduced from Ref. [94] with permission. Copyright 2018 American Chemical Society 


\section{Catalytic Applications of the Isolated Reactive Centers}

Developing prominently stable, efficient and low-cost catalysts is crucial for the practical applications in energy conversion systems. Advanced SACs have emerged as promising paradigms with excellent activity, selectivity and stability. In the current section, we select several recent successful cases to show the potential and capability of SACs in energy conversion driven by thermal, light and electric energy.

\subsection{Thermal Catalytic Reactions}

\subsubsection{CO Oxidation}

As a prototypical reaction in heterogeneous catalysis, $\mathrm{CO}$ oxidation has been extensively studied with variety of catalysts, especially Ru-, Au-, Pd- and Pt-based catalysts [98, 99]. However, the activity origin for the catalytic CO oxidation still hasn't been fully explored. The recently developed SACs not only enable $100 \%$ atomic utilization efficiency, but also offer a new approach to further understand the mechanism at the atomic level. Yan and colleagues have decorated the isolated $\mathrm{Pt}$ atoms onto the mesoporous $\mathrm{Al}_{2} \mathrm{O}_{3}$ $\left(\mathrm{Pt} / \mathrm{m}-\mathrm{Al}_{2} \mathrm{O}_{3}-\mathrm{O}_{2}\right)$ with varied loading contents [100]. The coordinately unsaturated $\mathrm{Al}^{3+}$ sites play a key role in anchoring $\mathrm{Pt}$ atoms via oxygen bridges. The as-synthesized hybrid $\mathrm{Pt} / \mathrm{m}-\mathrm{Al}_{2} \mathrm{O}_{3}-\mathrm{O}_{2}$ delivers excellent performance in the $\mathrm{CO}$ oxidation process (Fig. 6a-c). Encouragingly, the configuration of the isolated Pt centers, as well as the high catalytic performance, is well maintained in the long-term reaction period, which should be attributed to the mutual interactions between Pt and the support matrix.

Preferential oxidation of $\mathrm{CO}$ in $\mathrm{H}_{2}$-rich atmosphere is confirmed as the most direct and effective approach for removing $\mathrm{CO}$ and providing clean $\mathrm{H}_{2}$. Generally, oxidestabilized Au catalysts are highly effective for $\mathrm{CO}$ oxidation but less active for $\mathrm{H}_{2}$ oxidation at low temperature. Zhang and co-workers have decorated the isolated Au centers onto the $\mathrm{CeO}_{2}$ support with the loading content of 0.05 wt\%. (0.05 $\left.\mathrm{Au}_{1} / \mathrm{CeO}_{2}\right)$ [37]. X-ray absorption fine structure (XAFS) studies clearly demonstrate that the $\mathrm{Au}_{1}$ atoms are located at the Ce vacancy sites. The developed catalyst delivers high CO conversion (>99.5\%) in the presence of rich $\mathrm{H}_{2}$ at elevated temperatures. The authors attribute the exceptional catalytic performance to the obviously reduced capability of the $\mathrm{Au}_{1}$ SACs to dissociate $\mathrm{H}_{2}$ in the catalytic process. Meng and colleagues have also tracked the origin of the superior $\mathrm{CO}$ oxidation performance of the isolated $\mathrm{Pd}$ atoms decorated graphene (PdGr) [101]. Combining DFT simulations and micro-kinetics modeling, the authors have confirmed that the positively charged PdGr can bind strongly with $\mathrm{O}_{2}$, acting as the reactive species to convert $\mathrm{CO}$. The $\mathrm{CO}$ oxidation over the as-synthesized SAC mainly proceeds through revised Langmuir-Hinshelwood pathways, and the dissociation of the peroxide intermediate $(\mathrm{O}-\mathrm{O}-\mathrm{C}=\mathrm{O})$ is considered as the rate-limiting step.

\subsubsection{Water-gas Shift (WGS) Reaction}

WGS reaction is another crucial reaction in producing clean hydrogen and removing CO contamination for chemical processing, which also has been investigated on various supported catalysts. The related reaction mechanism is still under debate, especially for the real active sites in the catalytic process. Zhang and colleagues have anchored the isolated Ir atoms onto the $\mathrm{FeO}_{\mathrm{x}}$ support $\left(\mathrm{Ir}_{1} / \mathrm{FeO}_{x}\right)$ with rather low loading content $(0.01 \mathrm{wt} \%)$ [38]. The developed catalyst exhibits impressive catalytic activity for the WGS reaction. The related reaction rate can reach up to $43.4 \mathrm{~mol}$ $\mathrm{CO} \mathrm{g}_{\mathrm{Ir}}{ }^{-1} \mathrm{~h}^{-1}$ at $300^{\circ} \mathrm{C}$. This value is nearly 1 order of magnitude higher than its nanoparticle or cluster counterparts, even higher than the most active Pt- or Au-based catalysts. The authors believe that the isolated Ir atoms can obviously improve the reducibility of the $\mathrm{FeO}_{\mathrm{x}}$ support and thus generate amounts of oxygen vacancies, resulting in a greatly boosted catalytic activity for the WGS reaction.

Wang and coworkers have decorated isolated Pt atoms onto the $\mathrm{FeO}_{\mathrm{x}}$ support (Pt-SAC) for WGS reaction. The developed catalyst delivers greatly improved specific activity (nearly 10 times) when compared with the nanoparticles [102]. With the Pt loading content of $0.05 \mathrm{wt} \%$, Pt-SAC exhibits high CO conversion rate $(\sim 65 \%)$ at $300{ }^{\circ} \mathrm{C}$. Experimental results show that Pt-SAC possesses the lowest activation energy for the WGS reaction. The isolated $\mathrm{Pt}$ atoms can accelerate the generation of oxygen vacancies on $\mathrm{FeO}_{\mathrm{x}}$ and facilitate the dissociation of $\mathrm{H}_{2} \mathrm{O}$ to $\mathrm{H}_{2}$ and adsorbed $\mathrm{O}$. The adsorbed $\mathrm{O}$ can interact with the weakly adsorbed $\mathrm{CO}$ over the isolated $\mathrm{Pt}$ sites and then realize the generation of $\mathrm{CO}_{2}$. 
(a) $\mathrm{PtCl}_{6}{ }^{2-} \quad \mathrm{Al}\left(\mathrm{OCHCH}_{3} \mathrm{CH}_{3}\right)_{3}$
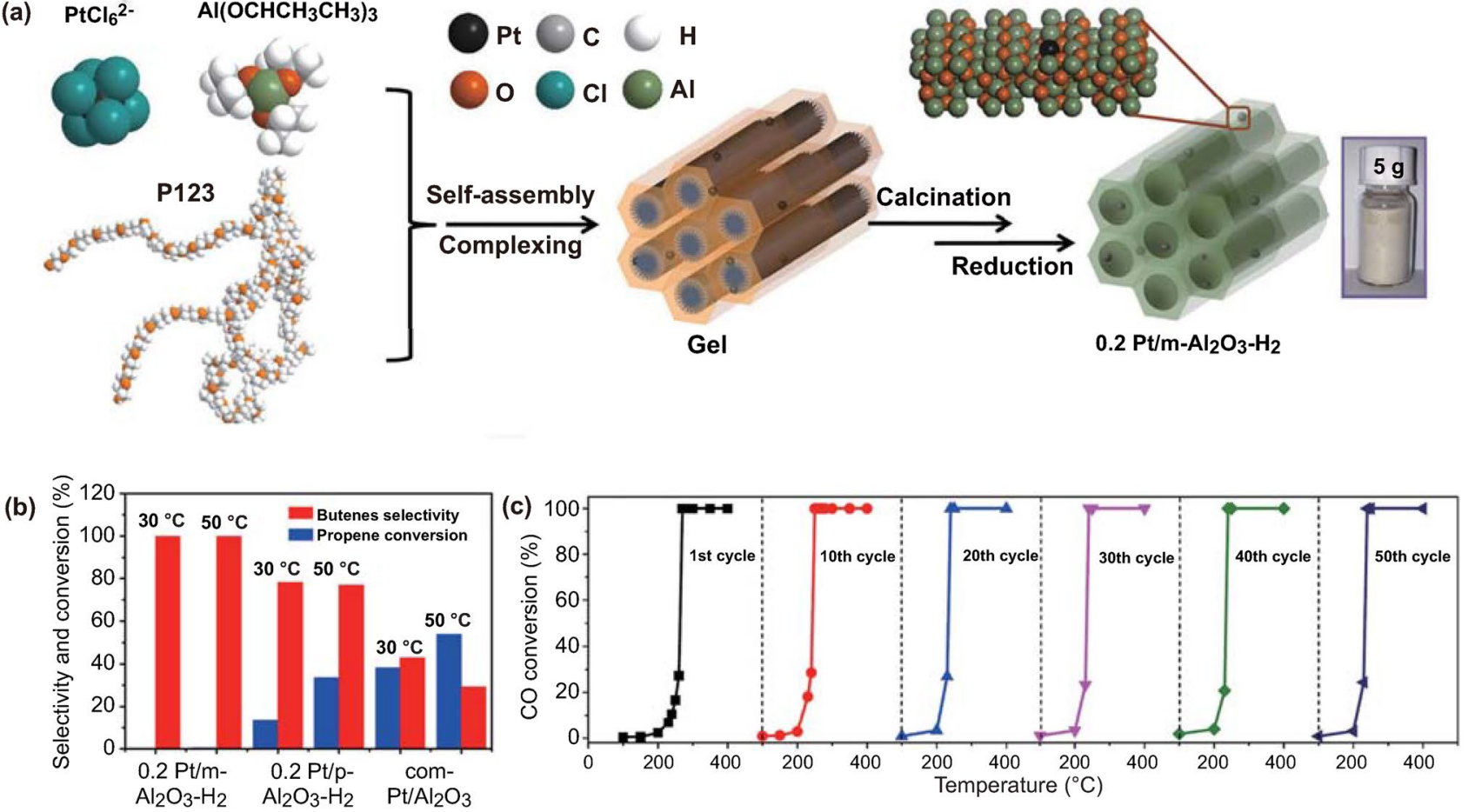

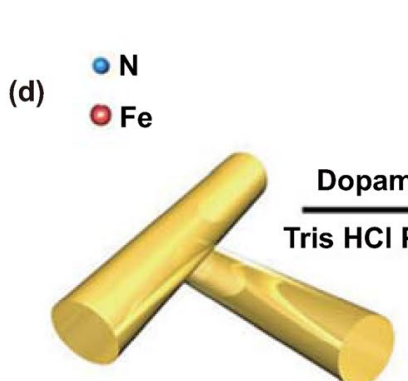

$\alpha-\mathrm{FeOOH}$ nanorod

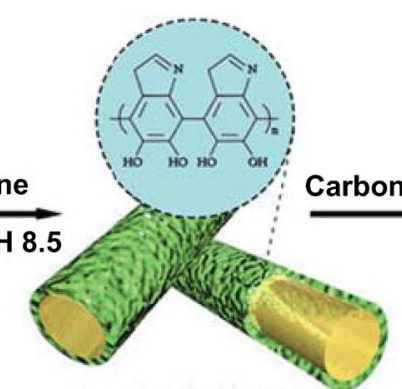

a-FeOOH@PDA

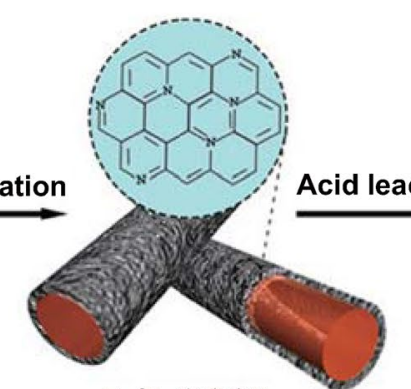

Fe/FeO@CN

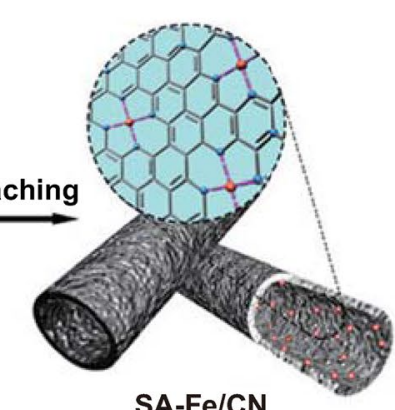

$\mathrm{SA}-\mathrm{Fe} / \mathrm{CN}$
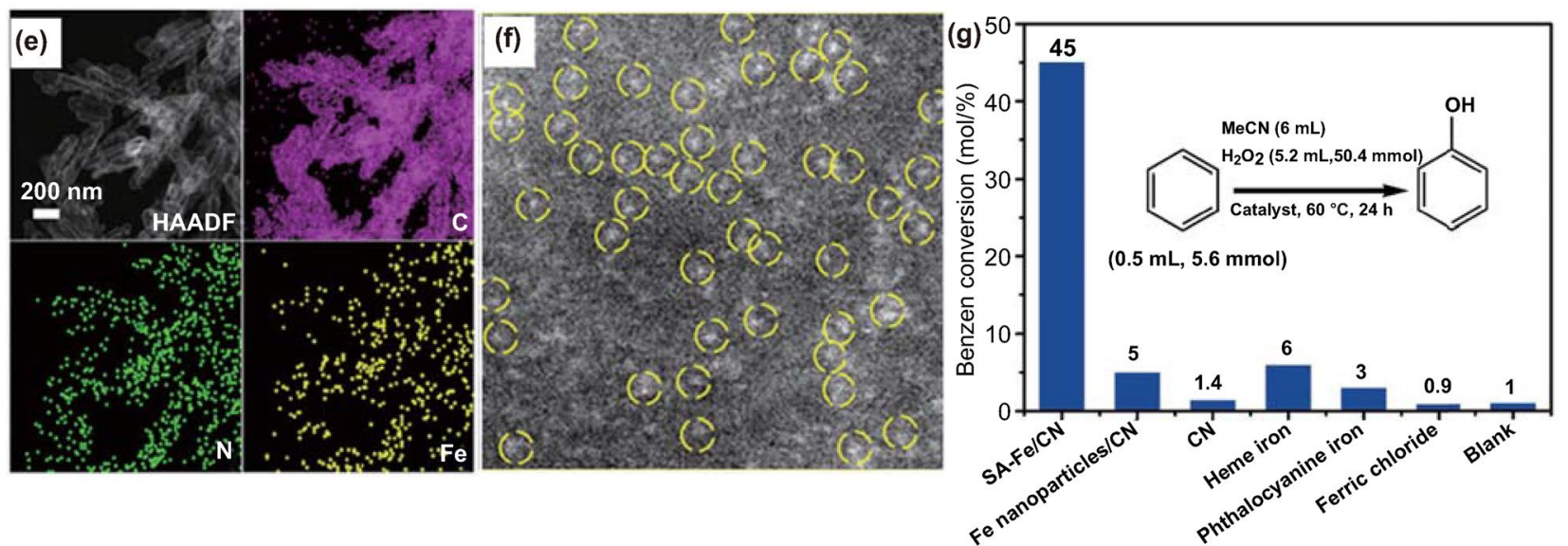

Fig. 6 a Schematic illustration of the $0.2 \mathrm{Pt} / \mathrm{m}-\mathrm{Al}_{2} \mathrm{O}_{3}-\mathrm{H}_{2}$ synthesis process. b The selectivity of butenes and conversion of propene at 30 and $50{ }^{\circ} \mathrm{C}$. c Conversion of $\mathrm{CO}$ from 100 to $400{ }^{\circ} \mathrm{C}$ with 1 st-50th cycles. a-c Reproduced from Ref. [100] with permission. Copyright 2017 Springer Nature. d Schematic Illustration of the Synthesis of SA-Fe/CN. e HAADF-STEM image and corresponding EDX mapping of SA-Fe/CN, C (pink), N (green) and Fe (yellow). f AC HAADF-STEM image of SA-Fe/CN. g Benzene conversion catalyzed by the SA-Fe/CN, Fe nanoparticles/CN, CN, heme iron, phthalocyanine iron and ferric chloride, respectively. d-g Reproduced from Ref. [107] with permission. Copyright 2017 American Chemical Society 


\subsubsection{Methane Conversion}

Direct conversion of natural gas into chemicals is an economical and eco-friendly approach. However, cleaving the first $\mathrm{C}-\mathrm{H}$ bond in $\mathrm{CH}_{4}$ is still a great challenge for the high bonding strength in $\mathrm{CH}_{4}$ molecule. Song and colleagues have decorated isolated chromium atoms on $\mathrm{TiO}_{2}$ support for direct methane oxidation [103]. The optimized catalyst exhibits an impressive yield of $57.9 \mathrm{~mol} / \mathrm{mol}_{\mathrm{Cr}}$ and high selectivity $(93 \%)$ at $50{ }^{\circ} \mathrm{C}$ for $\mathrm{C}_{1}$ oxygenated products. These values are among the highest values of most-recently reported catalysts. The quite good catalytic performance can be ascribed to the synergistic effect between isolated $\mathrm{Cr}$ atoms and $\mathrm{TiO}_{2}$ support. As proposed by the authors, the methane is firstly activated into the methyl radical, which is then converted into $\mathrm{CH}_{3} \mathrm{OH}$. The generated $\mathrm{CH}_{3} \mathrm{OH}$ is further oxidized to $\mathrm{HOCH}_{2} \mathrm{OOH}$ and $\mathrm{HCOOH}$. He and co-worker have further developed a bimetallic oxide cluster anions of $\mathrm{PtAl}_{2} \mathrm{O}_{4}{ }^{-}$to transform methane into formaldehyde with high selectivity under thermal collision conditions [104]. Mechanism investigations reveal that platinum atom rather than oxygen radical is the active species for $\mathrm{CH}_{4}$ activation at the beginning of the reaction. The $\mathrm{Al}_{2} \mathrm{O}_{4}{ }^{-}$support also plays a crucial role in the late stage of the reaction.

As one typical example, isolated iron atoms confined in the silica lattice have been reported with high catalytic selectivity for the nonoxidative conversion of methane to ethylene, aromatics and hydrogen [105]. During the reaction, $\mathrm{CH}_{4}$ can be activated on the isolated Fe sites to initially generate methyl radicals, followed by a series of gas-phase reactions. Wang and his colleagues have also synthesized the nanoceria-supported atomic Pt catalysts $\left(\mathrm{Pt}_{1} @ \mathrm{CeO}_{2}\right)$ and confirmed their advanced catalytic performance for the nonoxidative conversion of methane to $\mathrm{C}_{2}$ products (acetylene, ethane and ethylene) [106]. Mechanism investigations reveal that the isolated $\mathrm{Pt}$ atoms may be capable of stabilizing $\mathrm{C}_{2}$ adsorbates formed from the catalytic coupling of two dehydrogenated $\mathrm{C}_{1}$ adsorbates on the single Pt sites.

\subsubsection{Hydroxylation of Benzene}

Hydroxylation of benzene to phenol is a key reaction in chemical industry. Pursuing the generation of phenol with low energy input, high yield and selectivity is always the goal of scientific researchers. Thus, developing highefficiency and durable catalysts for the selective hydroxylation of benzene should be a crucial research topic. Li and colleagues have successfully confined the isolated $\mathrm{Fe}$ atoms onto the inner wall of a hollow carbon nanotube by employing a core-shell strategy (Fig. 6d-f) [107]. Impressive benzene conversion rate (45\%) and phenol selectivity (94\%) have been realized over the Fe-based SACs. These values are much higher than those of Fe nanoparticlebased catalysts (Fig. 6g). DFT simulation confirms that the exceptional activity should originate from the easier generation of activated oxygen species over the isolated Fe centers.

Wu and co-workers have also developed a cation-exchange strategy for decorating isolated metal atoms onto the edgerich nitrogen and sulfur co-doped graphene (SAC/S-N) [108]. They find that the $\mathrm{Cu}$ SAC/S-N is the most suitable catalyst for benzene oxidation reaction among all the synthesized SACs (such as $\mathrm{Pt}, \mathrm{Au}$, and $\mathrm{Ag}$ ), producing the high benzene conversion of $42.3 \%$ and a phenol selectivity of $93.4 \%$ at room temperature in $24 \mathrm{~h}$. DFT simulations suggest that edge-rich $\mathrm{S}, \mathrm{N}$ co-doped support could lower the reaction energy barrier of $\mathrm{Cu}$ sites, which greatly accelerates the thermodynamical process of the hydroxylation of benzene.

\subsection{Photocatalytic Reactions}

Photocatalytic reactions driven by solar energy play an imperative role in conquering the energy crisis and environmental pollution. Many photocatalytic reaction systems have been established in the past decades. However, the catalytic performances are still far from satisfactory because of the rapid electron-hole recombination and low active surface areas. SACs have received much attention in photocatalysis due to their maximal atomic utilization and fascinating strengths in enhancing light-harvesting, charge transfer dynamics and surface reactions [92, 109-112].

\subsubsection{Photocatalytic HER}

In 2014, Yang et al. have successfully deposited isolated metal atoms $(\mathrm{Pd}, \mathrm{Ru}, \mathrm{Pt}$, and $\mathrm{Rh})$ onto the $\mathrm{TiO}_{2}$ support as a model photocatalytic reaction system [113]. Those wellsynthesized SACs display superior photocatalytic hydrogen evolution activity when comparing to the counterpart metal 
(a)

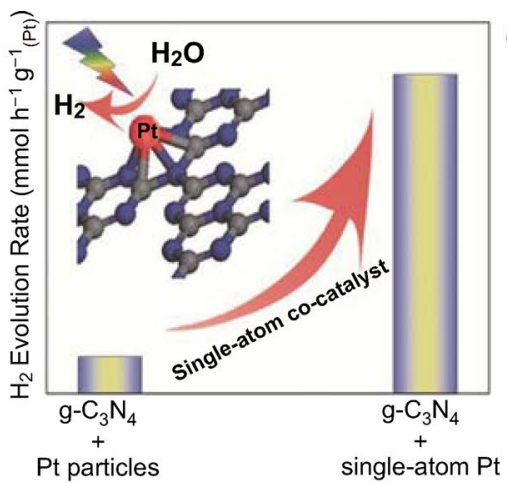

(b)

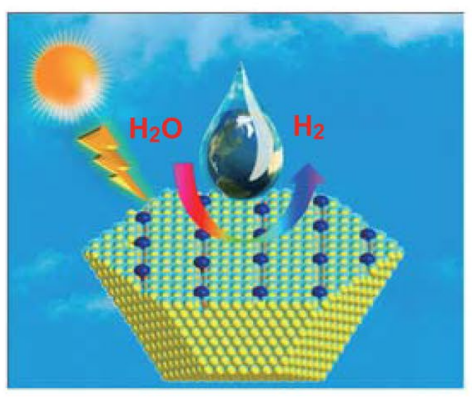

(c)

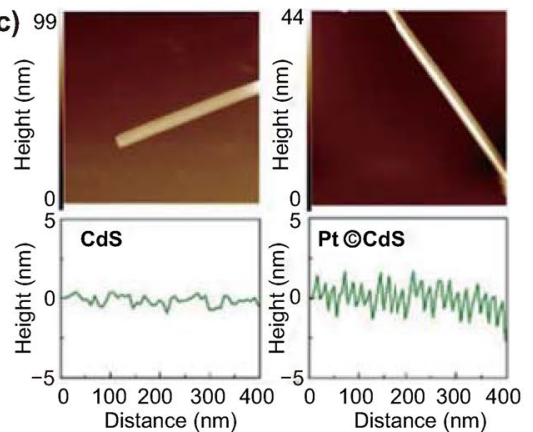

(d)

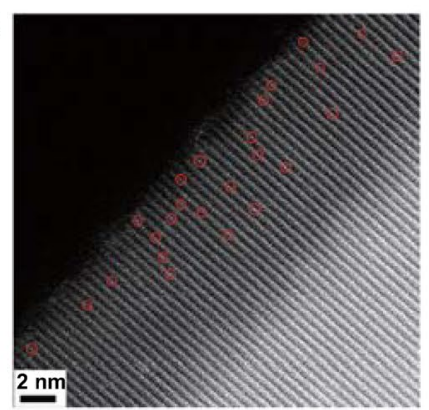

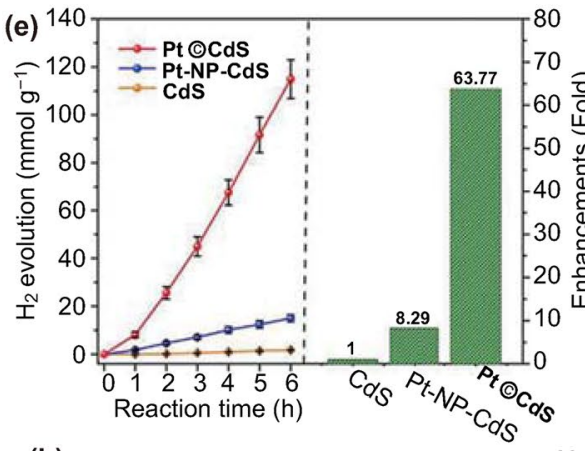

(h) 65

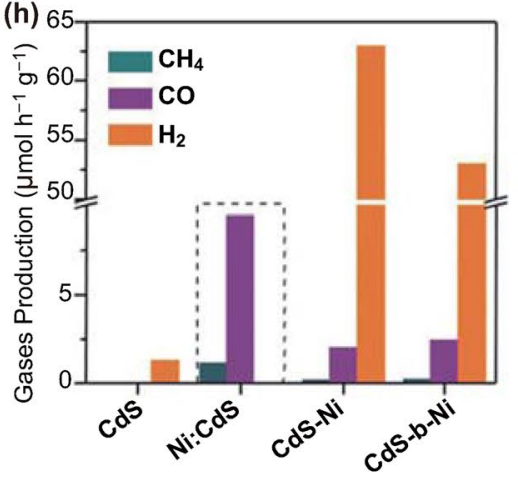

(f)

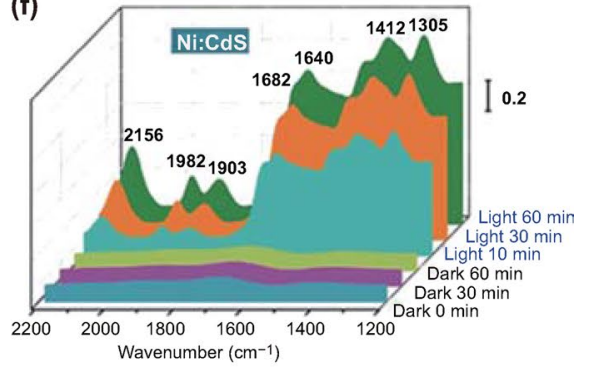

(g)

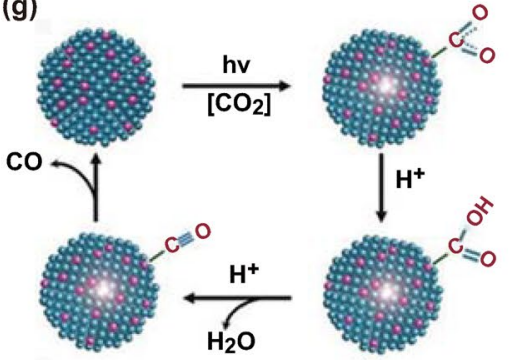

(j)

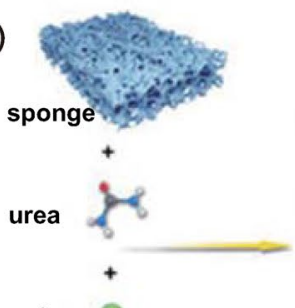

$\mathrm{Er}^{3+}$

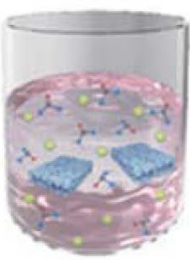

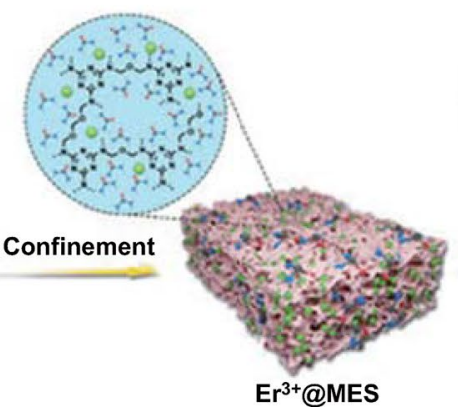
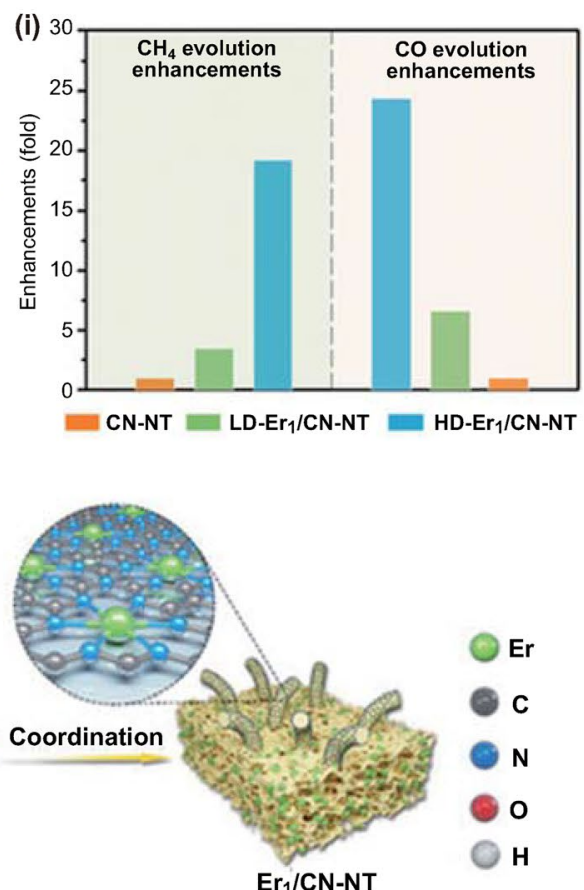

Fig. 7 a Pt-CN for photocatalytic $\mathrm{H}_{2}$ evolution. Reproduced from Ref. [114] with permission. Copyright 2016 WILEY-VCH Verlag GmbH \& Co. KGaA, Weinheim. b The geometric models of isolated platinum atoms decoration on the CdS surface with terraces for photocatalytic hydrogen evolution. c AFM maps and line scans for the CdS and Pt@CdS samples. d HAADF-STEM image in a spherical-aberration-corrected TEM for Pt@CdS. e Time dependent hydrogen evolution and enhancement of evolution over Pt@CdS (0.27 wt\% Pt loading), Pt-NP-CdS (0.25 wt $\%$ Pt loading) and CdS. b-e Reproduced from Ref. [115] with permission. Copyright 2017 Elsevier Ltd. f In situ DRIFTS spectra for the reaction of $\mathrm{CO}_{2}$ with $\mathrm{H}_{2} \mathrm{O}$ on $\mathrm{Ni}(0.26 \%)$ :CdS QDs under irradiation. $\mathbf{g}$ Proposed mechanism for the photocatalytic $\mathrm{CO}_{2}$ reduction at Ni:CdS QDs. h Average production rates of $\mathrm{CH}_{4}, \mathrm{CO}$, and $\mathrm{H}_{2}$ using various photocatalysts based on CdS QDs. f-h Reproduced from Ref. [116] with permission. Copyright 2018 Wiley-VCH Verlag GmbH \& Co. KGaA, Weinheim. i Enhancement of product evolution over the LD-Er $/$ CN-NT, HD-Er $/$ CN-NT, and CN-NT catalysts. $\mathbf{j}$ Schematic process of the synthesis of single-atom $\mathrm{Er}_{1} / \mathrm{CN}-\mathrm{NT}$ catalysts. i-j Reproduced from Ref. [118] with permission. Copyright 2020 Wiley-VCH Verlag GmbH \& Co. KGaA, Weinheim 
clusters on $\mathrm{TiO}_{2}$. Experimental results and DFT simulation suggest that the impressive photocatalytic hydrogen evolution performance of $\mathrm{TiO}_{2}$-supported isolated $\mathrm{Pt}$ atoms can be ascribed to the decreased $\mathrm{H}^{*}$ adsorption energy relative to that of metallic $\mathrm{Pt}$, which is closer to the optimum thermodynamically.

$\mathrm{Xie}$ and co-workers have decorated the isolated $\mathrm{Pt}$ atoms onto the $\mathrm{g}-\mathrm{C}_{3} \mathrm{~N}_{4}$ (Pt-CN) [114]. The prepared Pt-CN exhibits a prominently boosted activity for photocatalytic water splitting into hydrogen (Fig. 7a). The photo-excited electron transfer investigation suggests that the isolated $\mathrm{Pt}$ atoms can intrinsically change the surface trap states of $\mathrm{g}-\mathrm{C}_{3} \mathrm{~N}_{4}$, which should be the main reason for the dramatically boosted photocatalytic performance. Furthermore, Ye et al. have decorated the isolated $\mathrm{Pt}$ atoms onto the surface step of $\mathrm{CdS}$ nanowires (PtCCdS) (Fig. 7b-e) [115]. Pt@CdS exhibits a greatly improved photocatalytic performance for hydrogen evolution, which is 7.69 times higher than that of Pt nanoparticles and 63.77 times as high as the bare $\mathrm{CdS}$ nanowires. DFT simulations further suggest that the impressive catalytic activity should be ascribed to the decoration of positively charged $\mathrm{Pt}$ atoms with partially vacant $5 \mathrm{~d}$ orbitals. The existence of Pt atoms can greatly influence the charge density distribution and facilitate the transfer of the photo-generated electron.

\subsubsection{Photocatalytic $\mathrm{CO}_{2}$ Reduction Reaction}

The solar-driven $\mathrm{CO}_{2}$ reduction offers a highly important route to mitigate atmosphere $\mathrm{CO}_{2}$ emissions and convert solar energy into value-added chemicals. Amidst the numerous photocatalytic $\mathrm{CO}_{2}$ reduction systems in the past decade, SACs have exhibited great potential in achieving practical applications.

To overcome the limitations of low selectivity and stability of the established catalysts for $\mathrm{CO}_{2}$ reduction, Xiong et al. have doped isolated $\mathrm{Ni}$ atoms into the $\mathrm{CdS}$ quantum dots (Ni:CdS QDs) [116]. The obtained catalyst affords nearly $100 \%$ selectivity for the $\mathrm{CO}_{2}$ reduction into $\mathrm{CO}$ and $\mathrm{CH}_{4}$, a TON of approximately 35 in terms of the Ni atoms and excellent durability for more than $60 \mathrm{~h}$ (Fig. 7f-h). The doped isolated $\mathrm{Ni}$ atoms can trap the photo-excited electrons at surface catalytic sites and suppress the $\mathrm{H}_{2}$ evolution, playing a key role in the photocatalytic process. Liu and colleagues have further introduced isolated Co atom into
$\mathrm{Bi}_{3} \mathrm{O}_{4} \mathrm{Br}$ atomic layers [117]. The developed catalyst can deliver a high selectivity and activity in the light-driven $\mathrm{CO}_{2}$ reduction to $\mathrm{CO}$, about 32 and 4 times higher than those of bulk $\mathrm{Bi}_{3} \mathrm{O}_{4} \mathrm{Br}$ and atomic layer $\mathrm{Bi}_{3} \mathrm{O}_{4} \mathrm{Br}$, respectively. DFT simulation reveals that the isolated Co atoms in the $\mathrm{Bi}_{3} \mathrm{O}_{4} \mathrm{Br}$ can stabilize the $\mathrm{COOH}^{*}$ intermediates and modulate the rate-limiting step, resulting in greatly accelerated reaction kinetics.

Wang and coworkers have also decorated isolated rareearth single erbium (Er) atom onto the carbon nitride nanotubes $\left(\mathrm{Er}_{1} / \mathrm{CN}-\mathrm{NT}\right)$ with varied loading contents (Fig. 7i-j) [118]. The newly developed $\mathrm{Er}_{1} / \mathrm{CN}-\mathrm{NT}$ displays prominent photocatalytic $\mathrm{CO}_{2}$ reduction activity in a pure-water system. Systematic theoretical reaction mechanism simulations clearly reveal the favorable formation of gaseous $\mathrm{CO}$ than $\mathrm{CH}_{4}$ and unfavorable $\mathrm{H}_{2}$ production on the isolated rareearth Er atoms in $\mathrm{Er}_{1} / \mathrm{CN}-\mathrm{NT}$.

\subsection{Electrocatalytic Reactions}

\subsubsection{Electrocatalytic HER}

The electrochemical HER offers a clean and sustainable approach to produce $\mathrm{H}_{2}$ [119-124]. Pt-based catalysts have been regarded as the most active electrocatalysts to catalyze HER [125]. Bao and colleagues have decorated the isolated Pt atoms into $\mathrm{MoS}_{2}\left(\mathrm{Pt}-\mathrm{MoS}_{2}\right)$ via a one-pot chemical synthetic approach [126]. The inert in-plane $\mathrm{S}$ atoms of $\mathrm{MoS}_{2}$ can be greatly activated via the decoration of isolated $\mathrm{Pt}$ atoms, which is confirmed by the greatly improved HER performance. According to the DFT calculations, the activation of in-plane $\mathrm{MoS}_{2}$ should be directly reflected from the greatly changed adsorption behavior of $\mathrm{H}$ atoms over $\mathrm{S}$ atoms by neighboring doped Pt atoms (Fig. 8a, b).

Remarkable efforts have also been contributed to the development of efficient non-noble electrocatalysts as $\mathrm{Pt}$ alternatives [68, 127-129]. Liu and coworkers have decorated the isolated $\mathrm{Co}$ atoms into the lattice of the distorted $1 \mathrm{~T} \mathrm{MoS}_{2}$ nanosheets (SA Co-D $1 \mathrm{~T} \mathrm{MoS}_{2}$ ), which exhibits quite high long-term stability and impressive activity comparable to the Pt-based catalysts [130]. DFT simulation suggests that the impressive catalytic performance should be ascribed to the greatly modified hydrogen binding over the $\mathrm{S}$ atoms after the Co decoration. Tour and coworkers have incorporated trace amounts of individual Co atoms on the 
(a)

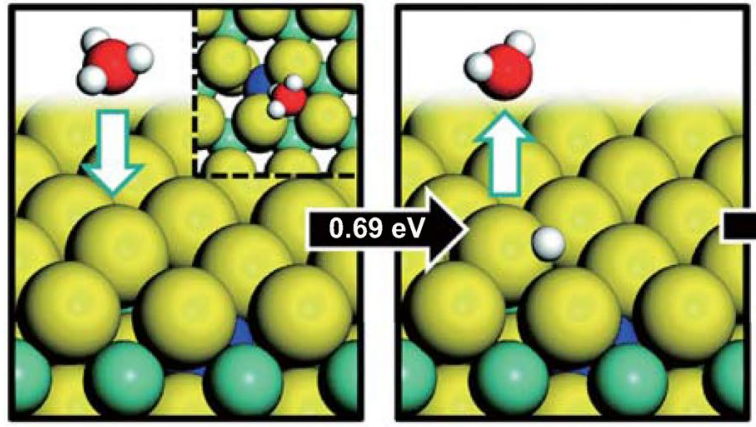

Volmer: $\mathrm{H}_{3} \mathrm{O}^{+}+\mathrm{e}^{-} \rightarrow \mathrm{H}_{\mathrm{ad}}+\mathrm{H}_{2} \mathrm{O}$

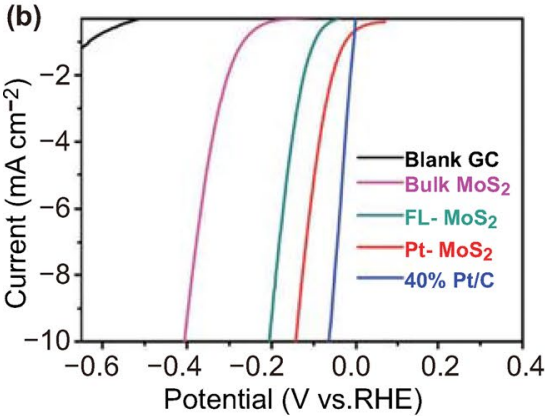

(c)

(e)

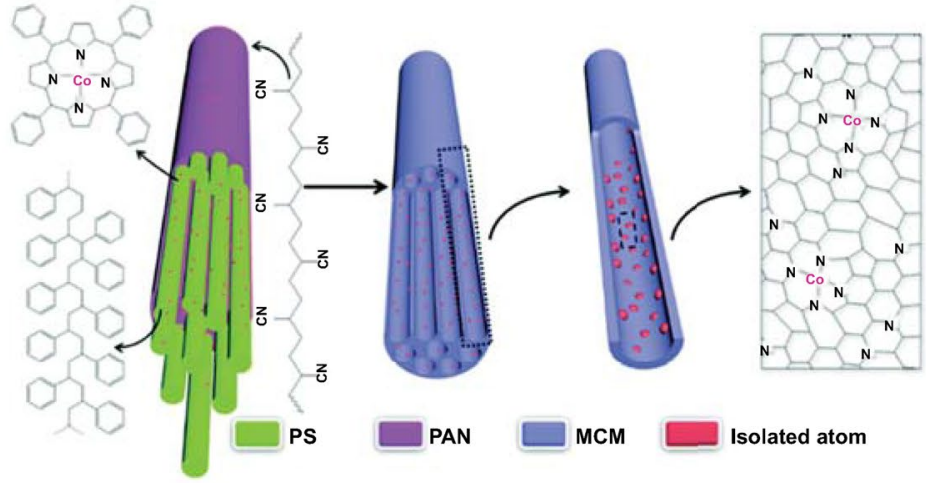

(g)

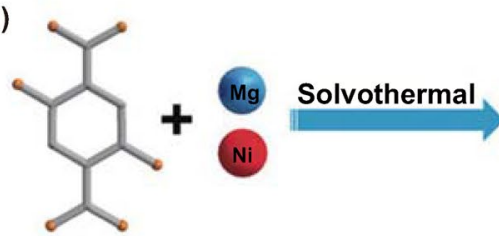

dobdc
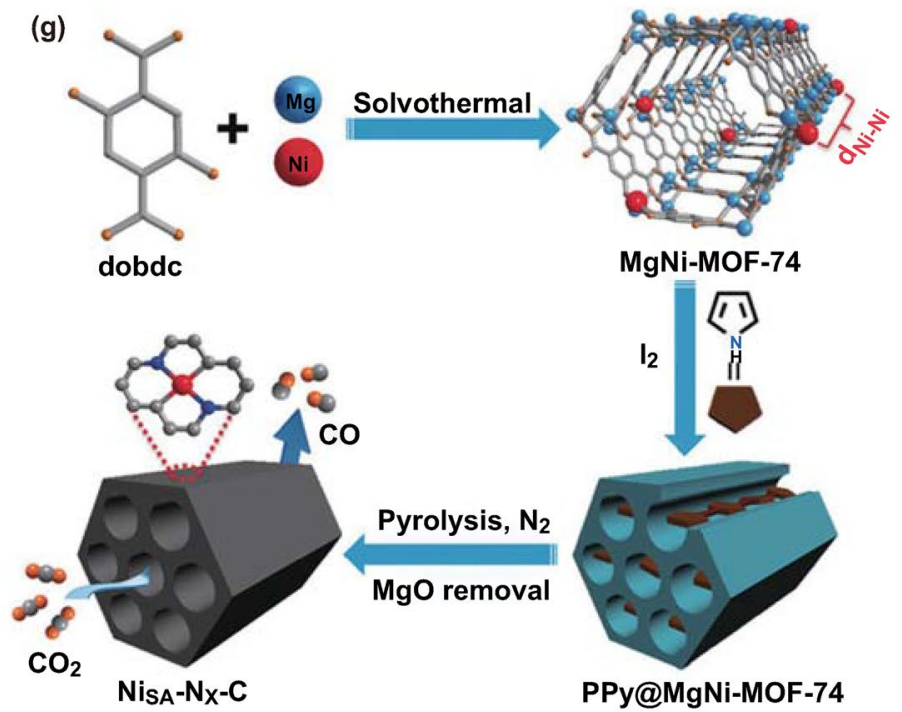

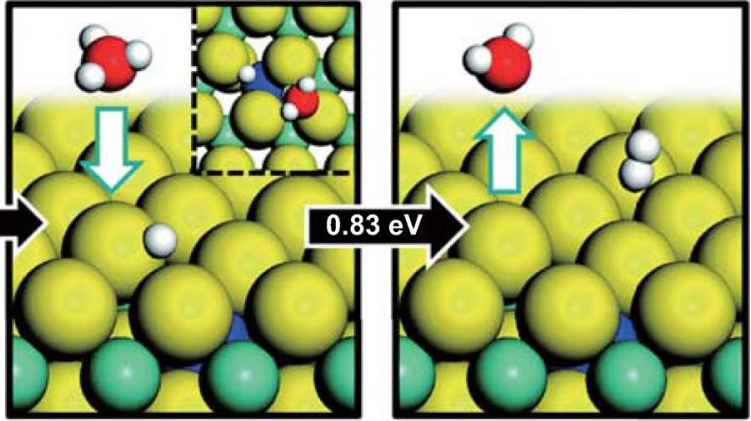

Heyrovsky: $\mathrm{H}_{3} \mathrm{O}^{+}+\mathrm{e}^{-}+\mathrm{H}_{\mathrm{ad}} \rightarrow \mathrm{H}_{2}+\mathrm{H}_{2} \mathrm{O}$
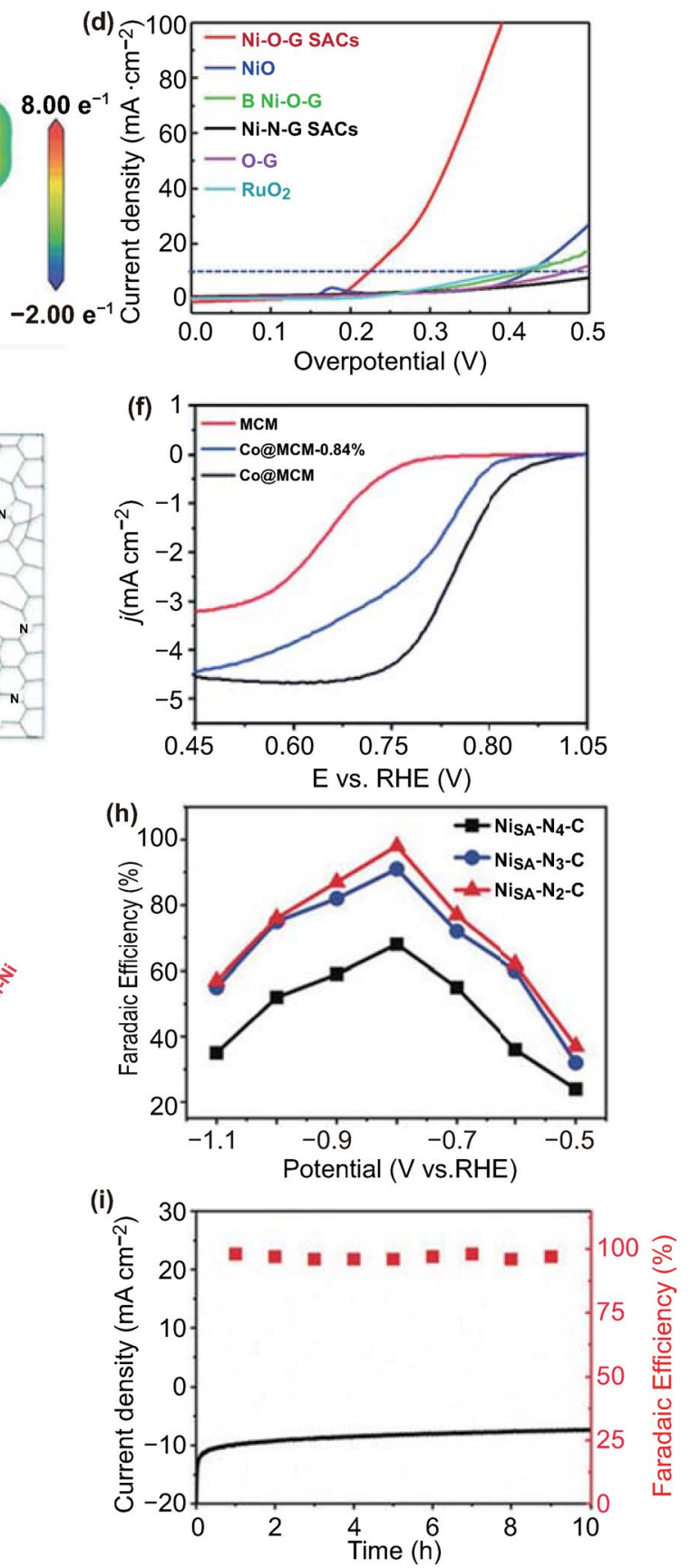
4Fig. 8 a HER process on a Pt-MoS 2 catalyst. The top views are shown in the insets. The reaction barriers are shown in the black arrows. The green, yellow, blue, red and white balls represent Mo, $\mathrm{S}, \mathrm{Pt}, \mathrm{O}$ and $\mathrm{H}$ atoms, respectively. b HER polarization curves for Pt- $\mathrm{MoS}_{2}$ in comparison with blank GC electrode, bulk $\mathrm{MoS}_{2}$, FL$\mathrm{MoS}_{2}$, and $40 \%$ Pt/C. a-b Reproduced from Ref. [126] with permission. Copyright 2015 The Royal Society of Chemistry. c Map of the DFT ESP surfaces of Ni-O-G SACs structure. Blue color indicates positive charges, and red color indicates negative charge. $\mathbf{d}$ The OER current curves of Ni-O-G SACs, NiO, B Ni-O-G, Ni-N-G SACs, $\mathrm{O}-\mathrm{G}$, and $\mathrm{RuO}_{2}$. c-d Reproduced from Ref. [135] with permission. Copyright 2020 The Authors. Published by WILEY-VCH Verlag $\mathrm{GmbH} \& \mathrm{Co}$. KGaA, Weinheim. e Schematic illustration of the synthetic process of Co@MCM. f ORR LSV curves of MCM, Co@ MCM-0.84\% and Co@MCM in $\mathrm{O}_{2}$-saturated $0.1 \mathrm{M} \mathrm{KOH}$ solution. e-f Reproduced from Ref. [156] with permission. Copyright 2018 The Royal Society of Chemistry. g Illustration showing the hostguest cooperative protection strategy for the fabrication of $\mathrm{Ni}_{\mathrm{SA}}-\mathrm{N}_{\mathrm{x}}-\mathrm{C}$ catalysts for electrocatalytic $\mathrm{CO}_{2}$ reduction. h FEs of $\mathrm{CO}$ at different applied potentials. i Stability of $\mathrm{Ni}_{\mathrm{SA}}-\mathrm{N}_{\mathrm{x}}-\mathrm{C}$ at $-0.8 \mathrm{~V}$ during $10 \mathrm{~h}$. g-i Reproduced from Ref. [161] with permission. Copyright 2019 Wiley-VCH Verlag GmbH \& Co. KGaA, Weinheim

nitrogen-doped graphene [131]. The identified Co atoms behaving as extraordinary active sites can minimize reaction kinetic barriers with a low onset potential of $\sim 30 \mathrm{mV}$ for hydrogen generation.

\subsubsection{Electrocatalytic Oxygen Evolution Reaction (OER)}

The high production of hydrogen through electrocatalytic water splitting is usually hindered by the kinetically sluggish OER. Therefore, highly efficient and robust SACs are urgently needed to enhance OER efficiency for minimizing the total overpotential for water splitting and other electronical applications [132-134]. Recently, Bao et al. have anchored individual high-valence nickel atoms on graphenelike carbon via oxygen sites (Ni-O-G SACs) [135]. The developed catalyst delivers impressive activity for OER. Low overpotential of $224 \mathrm{mV}$ is needed to realize the current density of $10 \mathrm{~mA} \mathrm{~cm}^{-2}$. Its Tafel slope $\left(42 \mathrm{mV} \mathrm{dec}^{-1}\right)$ and turnover frequency (TOF) of oxygen production at $300 \mathrm{mV}$ $\left(1.44 \mathrm{~S}^{-1}\right)$ have also been investigated and the obtained values are among the highest reported values for OER (Fig. 8c, d). The DFT simulations reveal that the highly oxidized Ni species in the configuration of $\mathrm{Ni}^{-} \mathrm{O}_{4}(\mathrm{OH})_{2}$ can reduce the Gibbs-free energy and extraordinarily boost OER performance of Ni-O-G SACs.

$\mathrm{Gu}$ and coworkers have fabricated isolated $\mathrm{Ir}$ atoms on $\mathrm{NiO}$ with ultrahigh loading content (18 wt\%) for OER [136]. The as-synthesized catalyst exhibits a 46 times increase in the OER current density compared with $\mathrm{IrO}_{2}$ at an overpotential of $260 \mathrm{mV}$. DFT calculations reveal that the substituted Ir atom can also improve the activity of surrounding $\mathrm{Ni}$ atoms. The increased catalytic sites and the cooperative interaction between isolated atoms and support are thereby responsible for the enhanced intrinsic activity.

\subsubsection{Electrocatalytic ORR}

ORR plays a crucial role in the energy storage and conversion systems such as fuel cells and some other renewable energy technologies [137-139]. So far, Pt and its alloys are typically regarded as the most efficient ORR catalysts [140-143]. Recently, Chou et al. have constructed a quasiPt-allotrope electrocatalyst (H-PtCo@ $\left.\mathrm{Pt}_{1} \mathrm{~N}-\mathrm{C}\right)$, composed of hollow $\mathrm{Pt}_{3} \mathrm{Co}$ alloy cores and isolated $\mathrm{Pt}$ atoms decorated $\mathrm{N}$-doped carbon matrix as shell [144]. This unique nanoarchitecture can prevent the agglomeration of hollow $\mathrm{Pt}_{3} \mathrm{Co}$ alloy ( $\mathrm{H}-\mathrm{PtCo})$ core and increase the ORR performance in virtue of the isolated $\mathrm{Pt}$ atoms. The $\mathrm{H}-\mathrm{PtCo} @ \mathrm{Pt}_{1} \mathrm{~N}-\mathrm{C}$ catalyst shows an efficient ORR activity in various organic electrolytes. Chen and coworkers have developed an Ir-N-C singleatom catalyst (Ir-SAC) to mimic homogeneous iridium porphyrins for high-efficiency ORR [145]. The as-synthesized Ir-SAC delivers orders of magnitude higher ORR activity than iridium nanoparticles. DFT simulations suggest that the obtained impressive performance should be ascribed to the optimized adsorption energy of reaction intermediates over the four nitrogen atoms coordinated iridium sites.

Tremendous attention has also been contributed to the fabrication of nonprecious-metal based ORR catalysts [146-154]. Bao and coworkers have confined isolated iron atoms via $\mathrm{N}$ atoms to form the $\mathrm{FeN}_{4}$ structure in the carbon matrix [155]. The developed catalyst delivers an impressive ORR activity, nearly comparable to the commercial $40 \% \mathrm{Pt} / \mathrm{C}$ catalyst. DFT simulations demonstrate that the nice ORR performance should be ascribed to the isolated Fe centers in the unsaturated configuration and high loading content. Lou and coworkers have decorated isolated cobalt atoms into a multichannel carbon matrix (Co@MCM) in the configuration of $\mathrm{CoN}_{4}$ (Fig. 8e) [156]. The unsaturated Co centers as well as the highly porous and conductive multichannel carbon substrate endow the catalyst with quite good ORR performance. DFT simulations verify that the barrier for the rate-determining step 
of $\mathrm{O}_{2} *$ reduction can be greatly reduced by the penetration of isolated Co sites (Fig. 8f).

\subsubsection{Electrocatalytic $\mathrm{CO}_{2}$ Reduction Reaction}

The electrocatalytic $\mathrm{CO}_{2}$ reduction into value-added products is an effective strategy to mitigate the energy crisis and related environmental issues [157, 158]. At present, the electrocatalytic $\mathrm{CO}_{2}$ reduction is faced with the large overpotential and a more complex reaction pathway than the above types of reactions due to the participation of various intermediates [159]. As a result, rational design of highly active and selective catalysts is important to regulate the $\mathrm{CO}_{2}$ reduction performance. Chen and colleagues have decorated the isolated $\mathrm{Pd}$ atoms into the nitrogen-doped carbon support for efficient electrocatalytic $\mathrm{CO}_{2}$ reduction [160]. This Pd SAC exhibits significantly improved $\mathrm{CO}_{2}$ reduction performance by avoiding the formation of $\mathrm{H}_{2}$. Further DFT calculation suggests that the $\mathrm{Pd}-\mathrm{N}_{4}$ site should be the real active sites for the $\mathrm{CO}$ evolution. The adsorbed $\mathrm{CO}_{2}$ intermediate can be stabilized in the $\mathrm{Pd}-\mathrm{N}_{4}$ sites and thus realize the electrocatalytic $\mathrm{CO}_{2}$ reduction at low overpotential.

Jiang et al. have introduced the isolated $\mathrm{Ni}$ atoms with controlled $\mathrm{Ni}-\mathrm{N}$ coordination number into the $\mathrm{N}$-doped carbon (Fig. 8g) [161]. Significantly, $\mathrm{Ni}_{\mathrm{SA}}-\mathrm{N}_{2}-\mathrm{C}$ featuring two nitrogen atoms coordinated $\mathrm{Ni}$ sites affords the best CO Faradaic efficiency (98\%) and turnover frequency $\left(1622 \mathrm{~h}^{-1}\right)$ among all the prepared Ni SACs in electrocatalytic $\mathrm{CO}_{2}$ reduction reaction (Fig. 8h, i). Theoretical calculations reveal that the unique electronic structure in $\mathrm{Ni}_{\mathrm{SA}^{-}}-\mathrm{N}_{2}-\mathrm{C}$ enables a facile formation of $\mathrm{COOH}^{*}$ intermediate and weak binding of $\mathrm{CO}$, thus giving rise to its superior performance. Xie and coworkers have also loaded the isolated Mo atoms on the ultrathin $\mathrm{N}$ doped graphene to promote the intrinsic catalytic activity of the substrate [162]. The prepared Mo SAC exhibits an improved $\mathrm{C}_{1}$ product selectivity and far better formate production rate with the aid of $4 \mathrm{~mol} \%$ ionic liquid than $\mathrm{N}$ doped graphene. DFT calculations reveal that the isolated Mo atoms can facilitate the preferred $\mathrm{CO}_{2}$ adsorption and the rate-determined reaction process, promoting the intrinsic electrocatalytic hydrogenation activity of $\mathrm{N}$ doped graphene.

\section{Conclusions and Outlook}

Significant breakthroughs for the development of SACs have been achieved in the past five years. It is very encouraging that we have developed various strategies for tailoring the local electronic structure and coordination environment of the isolated reactive sites. In the current review, we summarize and discuss the recent achievements in engineering the coordination sphere to explore the intrinsic activity of the SACs in heterogeneous catalysis. Our profound understanding of the SACs has been rapidly established in the terms of catalyst synthesis, catalytic application and mechanism exploration. Although it has been validated that SACs, as a prospective research frontier, present the ultra-most atomic utilization efficiency, successful paradigms for the practical application are still limited. Some foreseeable challenges and critical issues are urgent to be addressed to foster the rational design of more efficient SACs. Besides, possible solutions for addressing the existed challenges are also elaborated as below:

(I) Notwithstanding great progress has been realized in preparing the SACs, the synthesis of SACs with high loading content of isolated metal atoms is still an open question. Therefore, in-depth comprehending the synthetic processes of SACs and exploiting feasible strategies are vital to improve the density of active sites in SACs and implement high-performance catalytic reactions for desirable catalytic systems. Several approaches should be considered in the rational design of SACs with high loading density. Increasing the support area and introducing the defects and vacancies are beneficial for increasing the loading contents of the isolated atoms.

(II) As many catalytic processes are complicated with multi steps, the isolated active site may fail to satisfy the activation of all intermediates. To conquer this problem, developing multi-metal sites at atomic level is quite important. The recently developed atom by atom strategy for constructing the atomically precise metal clusters holds great promise for bridging the SACs and particle-based catalysts. Moreover, different metal species can produce varied activation effects in the catalytic process. The active centers can be generally modulated by the incorporation of varied metal elements. They may interact with each other and work in synergy to facilitate the catalytic kinetics for the particular catalysis. Research along this direction will 
offer new comprehension on the structure-activity correlations. The achievements would certainly benefit the whole heterogeneous catalysis community.

(III) The design of SACs at atomic level will generally challenge the limits of characterization. At present, the structural nature of SACs is mainly identified by the combination of XAFS and high-resolution electron microscopy techniques, which fail to provide the most accurate geometric and electronic structure of the reactive centers. For example, it is hard to tell the difference between the M-C and $\mathrm{M}-\mathrm{N}$ interactions since the scattering contributions of those coordinated atoms are nearly overlapped. Thus, developing atomically resolved electron microscopy techniques to distinguish different heavy metal as well as light elements is a continuous demand.

(IV) There is still a gap in the comprehension of catalytic kinetics and mechanism for the particular reaction types. With the help of operando characterization techniques, including the means of spectroscopic/ microscopic/imaging techniques, the structural evolution can be well tracked, which contributes to understanding the reaction mechanism of SACs under practical operating conditions. Thus, more attention should especially concern the operando characterizations to monitor the structure evolution of reactive centers as well as their interaction with main intermediates and final products. The obtained results can feedback clues about catalytic pathways and further provide a new perspective to design versatile SACs.

(V) Thanks to the uniformly distributed active sites, it is feasible to perform a theory-guided reaction simulation and thus provide us a platform for further understanding the catalytic mechanism. The progress in the big-data provides us additional assistants for simulating the binding strengths between active centers and reactants/intermediates/products, the reaction pathways, as well as the reaction barriers of some pivotal steps involved in the reaction process. The achieved structure-activity relationships can predict the catalytic performances for screening materials and finally establish an elegant platform for designing new catalysts for energy related applications.

(VI) Directly measuring the intrinsic catalytic activity of a single atom in real electrochemical environments is still challenging. Typically, most reported SACs are measured by depositing thin layers of catalyst on carbon or some other supports. The catalytic activity of the entire electrode is measured, and contribution from individual atoms is inferred rather than directly measured. Recently, microelectrochemical measurements have been becoming a mature technique to study the intrinsic activity of catalyst at atomistic resolution. Taking advantaging of the imaging of single atom at specific support (e.g. carbon, $\mathrm{MoS}_{2}$ ) by advanced microscopic imaging characterization, the intrinsic activity of single atom can be measured in microelectrochemical cells.

Acknowledgements This work is supported by NSFC (21773242, 21935010), the Strategic Priority Research Program of the Chinese Academy of Sciences (XDB20000000), National Key Research and Development Program of China (2018YFA0208600) and King Abdullah University of Science and Technology. J. Dong acknowledges financial support from Youth Innovation Promotion Association of Chinese Academy of Sciences (2018017).

Open Access This article is licensed under a Creative Commons Attribution 4.0 International License, which permits use, sharing, adaptation, distribution and reproduction in any medium or format, as long as you give appropriate credit to the original author(s) and the source, provide a link to the Creative Commons licence, and indicate if changes were made. The images or other third party material in this article are included in the article's Creative Commons licence, unless indicated otherwise in a credit line to the material. If material is not included in the article's Creative Commons licence and your intended use is not permitted by statutory regulation or exceeds the permitted use, you will need to obtain permission directly from the copyright holder. To view a copy of this licence, visit http://creativecommons.org/licenses/by/4.0/.

\section{References}

1. G.D. Sun, Z.J. Zhao, R.T. Mu, S.J. Zha, L.L. Li et al., Breaking the scaling relationship via thermally stable $\mathrm{Pt} / \mathrm{Cu}$ single atom alloys for catalytic dehydrogenation. Nat. Commun. 9, 4454 (2018). https://doi.org/10.1038/s41467-018-06967-8

2. X.F. Yang, A.Q. Wang, B.T. Qiao, J. Li, J.Y. Liu et al., Single-atom catalysts: A new frontier in heterogeneous catalysis. Acc. Chem. Res. 46(8), 1740-1748 (2013). https://doi.org/10. 1021/ar300361m

3. P.N. Duchesne, Z.Y. Li, C.P. Deming, V. Fung, X.J. Zhao et al., Golden single-atomic-site platinum electrocatalysts. Nat. Mater. 17(11), 1033-1039 (2018). https://doi.org/10. 1038/s41563-018-0167-5

4. H. Zhang, Y. Wang, S. Zuo, W. Zhou, J. Zhang et al., Isolated cobalt centers on $\mathrm{W}_{18} \mathrm{O}_{49}$ nanowires perform as a reaction switch for efficient $\mathrm{CO}_{2}$ photoreduction. J. Am. Chem. Soc. 
143, 2173-2177 (2021). https://doi.org/10.1021/jacs.0c084 09

5. H.L. Fei, J.C. Dong, Y.X. Feng, C.S. Allen, C.Z. Wan et al., General synthesis and definitive structural identification of $\mathrm{MN}_{4} \mathrm{C}_{4}$ single-atom catalysts with tunable electrocatalytic activities. Nat. Catal. 1(1), 63-72 (2018). https://doi.org/10. 1038/s41929-017-0008-y

6. X. Li, X. Yang, Y. Huang, T. Zhang, B. Liu, Supported noblemetal single atoms for heterogeneous catalysis. Adv. Mater. 31(50), e1902031 (2019). https://doi.org/10.1002/adma. 201902031

7. C.Z. Zhu, S.F. Fu, Q.R. Shi, D. Du, Y.H. Lin, Single-atom electrocatalysts. Angew. Chem. Int. Ed. 56(45), 13944-13960 (2017). https://doi.org/10.1002/anie.201703864

8. A.Q. Wang, J. Li, T. Zhang, Heterogeneous single-atom catalysis. Nat. Rev. Chem. 2(6), 65-81 (2018). https://doi. org/10.1038/s41570-018-0010-1

9. Y. Li, J. Li, J. Huang, J. Chen, Y. Kong et al., Boosting electroreduction kinetics of nitrogen to ammonia via tuning electron distribution of single-atomic iron sites. Angew. Chem. Int. Ed. 60(16), 9078-9085 (2021). https://doi.org/10.1002/ anie. 202100526

10. X. Wang, X. Sang, C.L. Dong, S. Yao, L. Shuai et al., Proton capture strategy for enhancing electrochemical $\mathrm{CO}_{2}$ reduction on atomically dispersed metal-nitrogen active sites. Angew. Chem. Int. Ed. (2021). https://doi.org/10.1002/anie.20210 0011

11. B.T. Qiao, A.Q. Wang, X.F. Yang, L.F. Allard, Z. Jiang et al., Single-atom catalysis of $\mathrm{CO}$ oxidation using $\mathrm{Pt}_{1} / \mathrm{FeO}_{\mathrm{x}}$. Nat. Chem. 3(8), 634-641 (2011). https://doi.org/10.1038/nchem. 1095

12. N.C. Cheng, S. Stambula, D. Wang, M.N. Banis, J. Liu et al., Platinum single-atom and cluster catalysis of the hydrogen evolution reaction. Nat. Commun. 7, 13638 (2016). https:// doi.org/10.1038/ncomms13638

13. H. Li, M. Wang, L. Luo, J. Zeng, Static regulation and dynamic evolution of single-atom catalysts in thermal catalytic reactions. Adv. Sci. 6(3), 1801471 (2019). https://doi. org/10.1002/advs.201801471

14. H. Zhang, X.F. Lu, Z.P. Wu, X.W.D. Lou, Emerging multifunctional single-atom catalysts/nanozymes. ACS Cent. Sci. 6(8), 1288-1301 (2020). https://doi.org/10.1021/acscentsci. 0c00512

15. E.D. Boyes, A.P. LaGrow, M.R. Ward, R.W. Mitchell, P.L. Gai, Single atom dynamics in chemical reactions. Acc. Chem. Res. 53(2), 390-399 (2020). https://doi.org/10.1021/acs. accounts.9b00500

16. Y.G. Yao, Z.N. Huang, P.F. Xie, L.P. Wu, L. Ma et al., High temperature shockwave stabilized single atoms. Nat. Nanotechnol. 14(9), 851-857 (2019). https://doi.org/10.1038/ s41565-019-0518-7

17. P. Zhou, F. Lv, N. Li, Y.L. Zhang, Z.J. Mu et al., Strengthening reactive metal-support interaction to stabilize high-density Pt single atoms on electron-deficient $\mathrm{g}_{-} \mathrm{C}_{3} \mathrm{~N}_{4}$ for boosting photocatalytic $\mathrm{H}_{2}$ production. Nano Energy 56, 127-137 (2019). https://doi.org/10.1016/j.nanoen.2018.11.033
18. W.H. Lai, L.F. Zhang, W.B. Hua, S. Indris, Z.C. Yan et al., General $\pi$-electron-assisted strategy for Ir, Pt, Ru, Pd, Fe, $\mathrm{Ni}$ single-atom electrocatalysts with bifunctional active sites for highly efficient water splitting. Angew. Chem. Int. Ed. 58(34), 11868-11873 (2019). https://doi.org/10.1002/ anie. 201904614

19. Q. Zhang, X.X. Qin, F.P. Duan-Mu, H.M. Ji, Z.R. Shen et al., Isolated platinum atoms stabilized by amorphous tungstenic acid: Metal-support interaction for synergistic oxygen activation. Angew. Chem. Int. Ed. 57(30), 93519356 (2018). https://doi.org/10.1002/anie.201804319

20. Y. Guo, S. Mei, K. Yuan, D.J. Wang, H.C. Liu et al., Lowtemperature $\mathrm{CO}_{2}$ methanation over $\mathrm{CeO}_{2}$-supported $\mathrm{Ru}$ single atoms, nanoclusters, and nanoparticles competitively tuned by strong metal-support interactions and $\mathrm{H}$-spillover effect. ACS Catal. 8(7), 6203-6215 (2018). https://doi.org/ 10.1021/acscatal.7b04469

21. S. Yang, Y.J. Tak, J. Kim, A. Soon, H. Lee, Support effects in single-atom platinum catalysts for electrochemical oxygen reduction. ACS Catal. 7(2), 1301-1307 (2017). https:// doi.org/10.1021/acscatal.6b02899

22. M. Moses-DeBusk, M. Yoon, L.F. Allard, D.R. Mullins, $\mathrm{Z}$. Wu et al., CO oxidation on supported single Pt atoms: experimental and ab initio density functional studies of $\mathrm{CO}$ interaction with $\mathrm{Pt}$ atom on $\theta-\mathrm{Al}_{2} \mathrm{O}_{3}(010)$ surface. J. Am. Chem. Soc. 135(34), 12634-12645 (2013). https://doi.org/ $10.1021 / \mathrm{ja} 401847 \mathrm{c}$

23. Z.W. Seh, J. Kibsgaard, C.F. Dickens, I.B. Chorkendorff, J.K. Norskov et al., Combining theory and experiment in electrocatalysis: Insights into materials design. Science 355(6321), eaad4998 (2017). https://doi.org/10.1126/scien ce.aad4998

24. J.D. Benck, T.R. Hellstern, J. Kibsgaard, P. Chakthranont, T.F. Jaramillo, Catalyzing the hydrogen evolution reaction (HER) with molybdenum sulfide nanomaterials. ACS Catal. 4(11), 3957-3971 (2014). https://doi.org/10.1021/cs500923c

25. M. Zhou, S.J. Bao, A.J. Bard, Probing size and substrate effects on the hydrogen evolution reaction by single isolated $\mathrm{Pt}$ atoms, atomic clusters, and nanoparticles. J. Am. Chem. Soc. 141(18), 7327-7332 (2019). https://doi.org/10.1021/ jacs.8b13366

26. H. Wang, J.X. Liu, L.F. Allard, S. Lee, J.L. Liu et al., Surpassing the single-atom catalytic activity limit through paired Pt-O-Pt ensemble built from isolated $\mathrm{Pt}_{1}$ atoms. Nat. Commun. 10, 3808 (2019). https://doi.org/10.1038/ s41467-019-11856-9

27. S. Kawai, A.S. Foster, T. Bjorkman, S. Nowakowska, J. Bjork et al., Van der Waals interactions and the limits of isolated atom models at interfaces. Nat. Commun. 7, 11559 (2016). https://doi.org/10.1038/ncomms11559

28. G. Kyriakou, M.B. Boucher, A.D. Jewell, E.A. Lewis, T.J. Lawton et al., Isolated metal atom geometries as a strategy for selective heterogeneous hydrogenations. Science 335(6073), 1209-1212 (2012). https://doi.org/10.1126/science.1215864

29. Y. Wang, F.L. Hu, Y. Mi, C. Yan, S. Zhao, Single-metal-atom catalysts: An emerging platform for electrocatalytic oxygen 
reduction. Chem. Eng. J. 406, 127135 (2021). https://doi.org/ 10.1016/j.cej.2020.127135

30. J. Wang, Z. Li, Y. Wu, Y. Li, Fabrication of single-atom catalysts with precise structure and high metal loading. Adv. Mater. 30, 1801649 (2018). https://doi.org/10.1002/adma. 201801649

31. Z. Pu, I.S. Amiinu, R. Cheng, P. Wang, C. Zhang et al., Single-atom catalysts for electrochemical hydrogen evolution reaction: Recent advances and future perspectives. Nano-Micro Lett. 12(1), 21 (2020). https://doi.org/10.1007/ s40820-019-0349-y

32. B. Wang, H. Cai, S. Shen, Single metal atom photocatalysis. Small Method 3(9), 1800447 (2019). https://doi.org/10.1002/ smtd.201800447

33. L. Nie, D.H. Mei, H.F. Xiong, B. Peng, Z.B. Ren et al., Activation of surface lattice oxygen in single-atom $\mathrm{Pt} / \mathrm{CeO}_{2}$ for low-temperature CO oxidation. Science 358(6369), 14191423 (2017). https://doi.org/10.1126/science.aao2109

34. L.L. Lin, W. Zhou, R. Gao, S.Y. Yao, X. Zhang et al., Lowtemperature hydrogen production from water and methanol using Pt/ $\alpha-$ MoC catalysts. Nature 544(7648), 80-83 (2017). https://doi.org/10.1038/nature21672

35. Y. Yang, Y. Yang, Z. Pei, K.H. Wu, C. Tan et al., Recent progress of carbon-supported single-atom catalysts for energy conversion and storage. Matter 3(5), 1442-1476 (2020). https://doi.org/10.1016/j.matt.2020.07.032

36. Y. Wang, K. Qi, S. Yu, G. Jia, Z. Cheng et al., Revealing the intrinsic peroxidase-like catalytic mechanism of heterogeneous single-atom Co-MoS 2 . Nano-Micro Lett. 11(1), 102 (2019). https://doi.org/10.1007/s40820-019-0324-7

37. B.T. Qiao, J.X. Liu, Y.G. Wang, Q.Q. Lin, X.Y. Liu et al., Highly efficient catalysis of preferential oxidation of $\mathrm{CO}$ in $\mathrm{H}_{2}$-rich stream by gold single-atom catalysts. ACS Catal. 5(11), 6249-6254 (2015). https://doi.org/10.1021/acscatal. $5 \mathrm{~b} 01114$

38. J. Lin, A.Q. Wang, B.T. Qiao, X.Y. Liu, X.F. Yang et al., Remarkable performance of $\operatorname{Ir}_{1} / \mathrm{FeO}_{\mathrm{x}}$ single-atom catalyst in water gas shift reaction. J. Am. Chem. Soc. 135(41), 1531415317 (2013). https://doi.org/10.1021/ja408574m

39. H.J. Zhang, T. Watanabe, M. Okumura, M. Haruta, N. Toshima, Catalytically highly active top gold atom on palladium nanocluster. Nat. Mater. 11(1), 49-52 (2012). https:// doi.org/10.1038/nmat3143

40. G.X. Pei, X.Y. Liu, A.Q. Wang, A.F. Lee, M.A. Isaacs et al., Ag alloyed Pd single-atom catalysts for efficient selective hydrogenation of acetylene to ethylene in excess ethylene. ACS Catal. 5(6), 3717-3725 (2015). https://doi.org/10.1021/ acscatal.5b00700

41. P. Liu, Y. Zhao, R. Qin, S. Mo, G. Chen et al., Photochemical route for synthesizing atomically dispersed palladium catalysts. Science 352(6287), 797-801 (2016). https://doi.org/10. 1126/science.aaf5251

42. Z. Liang, C. Qu, W. Guo, R. Zou, Q. Xu, Pristine metalorganic frameworks and their composites for energy storage and conversion. Adv. Mater. 30(37), e1702891 (2018). https://doi.org/10.1002/adma.201702891
43. Y.F. Gu, Y.N. Wu, L.C. Li, W. Chen, F.T. Li et al., Controllable modular growth of hierarchical MOF-on-MOF architectures. Angew. Chem. Int. Ed. 56(49), 15658-15662 (2017). https://doi.org/10.1002/anie.201709738

44. H.X. Zhang, F. Wang, H. Yang, Y.X. Tan, J. Zhang et al., Interrupted zeolite LTA and ATN-type boron imidazolate frameworks. J. Am. Chem. Soc. 133(31), 11884-11887 (2011). https://doi.org/10.1021/ja2040927

45. J.R. Li, R.J. Kuppler, H.C. Zhou, Selective gas adsorption and separation in metal-organic frameworks. Chem. Soc. Rev. 38(5), 1477-1504 (2009). https://doi.org/10.1039/ B802426J

46. G. Xu, H. Zhang, J. Wei, H.X. Zhang, X. Wu et al., Integrating the $\mathrm{g}-\mathrm{C}_{3} \mathrm{~N}_{4}$ nanosheet with $\mathrm{B}-\mathrm{H}$ bonding decorated metal-organic framework for $\mathrm{CO}_{2}$ activation and photoreduction. ACS Nano 12, 5333-5340 (2018). https://doi.org/10. 1021/acsnano.8b00110

47. Y. Zhang, L. Jiao, W. Yang, C. Xie, H.L. Jiang, Rational fabrication of low-coordinate single-atom $\mathrm{Ni}$ electrocatalysts by MOFs for highly selective $\mathrm{CO}_{2}$ reduction. Angew. Chem. Int. Ed. 60, 7607-7611 (2021). https://doi.org/10.1002/anie. 202016219

48. L. Jiao, W. Yang, G. Wan, R. Zhang, X. Zheng et al., Singleatom electrocatalysts from multivariate metal-organic frameworks for highly selective reduction of $\mathrm{CO}_{2}$ at low pressures. Angew. Chem. Int. Ed. 59, 20589-20595 (2020). https://doi. org/10.1002/anie.202008787

49. L. Jiao, R. Zhang, G. Wan, W. Yang, X. Wan et al., Nanocasting $\mathrm{SiO}_{2}$ into metal-organic frameworks imparts dual protection to high-loading Fe single-atom electrocatalysts. Nat. Commun. 11, 2831 (2020). https://doi.org/10.1038/ s41467-020-16715-6

50. H. Zhang, J. Wei, J. Dong, G. Liu, L. Shi et al., Efficient visible-light-driven carbon dioxide reduction by a single-atom implanted metal-organic framework. Angew. Chem. Int. Ed. 55(46), 14310-14314 (2016). https://doi.org/10.1002/anie. 201608597

51. X. Feng, Y. Song, Z. Li, M. Kaufmann, Y. Pi et al., Metalorganic framework stabilizes a low-coordinate iridium complex for catalytic methane borylation. J. Am. Chem. Soc. 141(28), 11196-11203 (2019). https://doi.org/10.1021/jacs. $9 \mathrm{~b} 04285$

52. X. Wang, W.X. Chen, L. Zhang, T. Yao, W. Liu et al., Uncoordinated amine groups of metal-organic frameworks to anchor single $\mathrm{Ru}$ sites as chemoselective catalysts toward the hydrogenation of quinoline. J. Am. Chem. Soc. 139(28), 9419-9422 (2017). https://doi.org/10.1021/jacs.7b01686

53. X.Z. Fang, Q.C. Shang, Y. Wang, L. Jiao, T. Yao et al., Single Pt atoms confined into a metal-organic framework for efficient photocatalysis. Adv. Mater. 30(7), 1705112 (2018). https://doi.org/10.1002/adma.201705112

54. P.Q. Yin, T. Yao, Y. Wu, L.R. Zheng, Y. Lin et al., Single cobalt atoms with precise $\mathrm{N}$-coordination as superior oxygen reduction reaction catalysts. Angew. Chem. Int. Ed. 55(36), 10800-10805 (2016). https://doi.org/10.1002/anie.20160 4802 
55. C.M. Zhao, X.Y. Dai, T. Yao, W.X. Chen, X.Q. Wang et al., Ionic exchange of metal organic frameworks to access single nickel sites for efficient electroreduction of $\mathrm{CO}_{2}$. J. Am. Chem. Soc. 139(24), 8078-8081 (2017). https://doi.org/10. 1021/jacs.7b02736

56. J. Wang, Z.Q. Huang, W. Liu, C.R. Chang, H.L. Tang et al., Design of N-coordinated dual-metal sites: A stable and active Pt-free catalyst for acidic oxygen reduction reaction. J. Am. Chem. Soc. 139(48), 17281-17284 (2017). https:// doi.org/10.1021/jacs.7b10385

57. Y. Li, R. Zhang, W. Zhou, X. Wu, H. Zhang et al., Hierarchical $\mathrm{MoS}_{2}$ hollow architectures with abundant Mo vacancies for efficient sodium storage. ACS Nano 13, 5533-5540 (2019). https://doi.org/10.1021/acsnano.9b00383

58. Y. Li, S. Zuo, Q.H. Li, X. Wu, J. Zhang et al., Vertically aligned $\mathrm{MoS}_{2}$ with in-plane selectively cleaved Mo-S bond for hydrogen production. Nano Lett. 21, 1848-1855 (2021). https://doi.org/10.1021/acs.nanolett.0c04978

59. F. Dvorak, M.F. Camellone, A. Tovt, N.D. Tran, F.R. Negreiros et al., Creating single-atom Pt-ceria catalysts by surface step decoration. Nat. Commun. 7, 10801 (2016). https://doi.org/10.1038/ncomms 10801

60. B. Zhang, H. Asakura, J. Zhang, J. Zhang, S. De et al., Stabilizing a platinum 1 single-atom catalyst on supported phosphomolybdic acid without compromising hydrogenation activity. Angew. Chem. Int. Ed. 55(29), 8319-8323 (2016). https://doi.org/10.1002/anie.201602801

61. Y.J. Chen, S.F. Ji, W.M. Sun, Y.P. Lei, Q.C. Wang et al., Engineering the atomic interface with single platinum atoms for enhanced photocatalytic hydrogen production. Angew. Chem. Int. Ed. 59(3), 1295-1301 (2020). https:// doi.org/10.1002/anie.201912439

62. S.H. Sun, G.X. Zhang, N. Gauquelin, N. Chen, J.G. Zhou et al., Single-atom catalysis using Pt/graphene achieved through atomic layer deposition. Sci. Rep. 3, 1775 (2013). https://doi.org/10.1038/srep01775

63. Y. Wang, J. Mao, X.G. Meng, L. Yu, D.H. Deng et al., Catalysis with two-dimensional materials confining single atoms: concept, design, and applications. Chem. Rev. 119(3), 1806-1854 (2019). https://doi.org/10.1021/acs. chemrev.8b00501

64. X.J. Cui, J.P. Xiao, Y.H. Wu, P.P. Du, R. Si et al., A graphene composite material with single cobalt active sites: A highly efficient counter electrode for dye-sensitized solar cells. Angew. Chem. Int. Ed. 55(23), 6708-6712 (2016). https:// doi.org/10.1002/anie.201602097

65. D.H. Deng, X.Q. Chen, L. Yu, X. Wu, Q.F. Liu et al., A single iron site confined in a graphene matrix for the catalytic oxidation of benzene at room temperature. Sci. Adv. 1(11), e1500462 (2015). https://doi.org/10.1126/sciadv.1500462

66. J. Jones, H.F. Xiong, A.T. Delariva, E.J. Peterson, H. Pham et al., Thermally stable single-atom platinum-on-ceria catalysts via atom trapping. Science 353(6295), 150-154 (2016). https://doi.org/10.1126/science.aaf8800

67. S.J. Wei, A. Li, J.C. Liu, Z. Li, W.X. Chen et al., Direct observation of noble metal nanoparticles transforming to thermally stable single atoms. Nat. Nanotechnol. 13(9), 856-861 (2018). https://doi.org/10.1038/s41565-018-0197-9

68. W. Chen, J. Pei, C.T. He, J. Wan, H. Ren et al., Single tungsten atoms supported on MOF-derived N-doped carbon for robust electrochemical hydrogen evolution. Adv. Mater. 30(30), e1800396 (2018). https://doi.org/10.1002/adma. 201800396

69. G. Vile, D. Albani, M. Nachtegaal, Z. Chen, D. Dontsova et al., A stable single-site palladium catalyst for hydrogenations. Angew. Chem. Int. Ed. 54(38), 11265-11269 (2015). https://doi.org/10.1002/anie.201505073

70. W. Wan, C.A. Triana, J. Lan, J. Li, C.S. Allen et al., Bifunctional single atom electrocatalysts: Coordination-performance correlations and reaction pathways. ACS Nano 14(10), 13279-13293 (2020). https://doi.org/10.1021/acsnano.0c050 88

71. H. Zhang, Y. Liu, T. Chen, J. Zhang, J. Zhang et al., Unveiling the activity origin of electrocatalytic oxygen evolution over isolated $\mathrm{Ni}$ atoms supported on a $\mathrm{N}$-doped carbon matrix. Adv. Mater. 31(48), e1904548 (2019). https://doi.org/10. 1002/adma.201904548

72. Y. Han, Y. Wang, R. Xu, W. Chen, L. Zheng et al., Electronic structure engineering to boost oxygen reduction activity by controlling the coordination of the central metal. Energy Environ. Sci. 11(9), 2348-2352 (2018). https://doi.org/10. 1039/c8ee01481g

73. W. Liu, L. Zhang, X. Liu, X. Liu, X. Yang et al., Discriminating catalytically active $\mathrm{FeN}_{\mathrm{x}}$ species of atomically dispersed $\mathrm{Fe}-\mathrm{N}-\mathrm{C}$ catalyst for selective oxidation of the $\mathrm{C}-\mathrm{H}$ bond. J. Am. Chem. Soc. 139(31), 10790-10798 (2017). https://doi. org/10.1021/jacs.7b05130

74. Z. Chen, W. Gong, Z. Liu, S. Cong, Z. Zheng et al., Coordination-controlled single-atom tungsten as a non-3d-metal oxygen reduction reaction electrocatalyst with ultrahigh mass activity. Nano Energy 60, 394-403 (2019). https://doi.org/10. 1016/j.nanoen.2019.03.045

75. Z. Jakub, J. Hulva, M. Meier, R. Bliem, F. Kraushofer et al., Local structure and coordination define adsorption in a model $\mathrm{Ir}_{1} / \mathrm{Fe}_{3} \mathrm{O}_{4}$ single-atom catalyst. Angew. Chem. Int. Ed. 58(39), 13961-13968 (2019). https://doi.org/10.1002/anie.20190 7536

76. Y. Xu, M. Chu, F. Liu, X. Wang, Y. Liu et al., Revealing the correlation between catalytic selectivity and the local coordination environment of Pt single atom. Nano Lett. 20(9), 6865-6872 (2020). https://doi.org/10.1021/acs.nanolett. 0c02940

77. H. Shen, E. Gracia-Espino, J. Ma, K. Zang, J. Luo et al., Synergistic effects between atomically dispersed $\mathrm{Fe}-\mathrm{N}-\mathrm{C}$ and $\mathrm{C}-\mathrm{S}-\mathrm{C}$ for the oxygen reduction reaction in acidic media. Angew. Chem. Int. Ed. 56(44), 13800-13804 (2017). https:// doi.org/10.1002/anie.201706602

78. Y. Mun, S. Lee, K. Kim, S. Kim, S. Lee et al., Versatile strategy for tuning ORR activity of a single $\mathrm{Fe}-\mathrm{N}_{4}$ site by controlling electron-withdrawing/donating properties of a carbon plane. J. Am. Chem. Soc. 141(15), 6254-6262 (2019). https:// doi.org/10.1021/jacs.8b13543 
79. Y. Chen, S. Ji, S. Zhao, W. Chen, J. Dong et al., Enhanced oxygen reduction with single-atomic-site iron catalysts for a zinc-air battery and hydrogen-air fuel cell. Nat. Commun. 9(1), 5422 (2018). https://doi.org/10.1038/ s41467-018-07850-2

80. Z. Luo, H. Zhang, Y. Yang, X. Wang, Y. Li et al., Reactant friendly hydrogen evolution interface based on di-anionic $\mathrm{MoS}_{2}$ surface. Nat. Commun. 11(1), 1116 (2020). https:// doi.org/10.1038/s41467-020-14980-z

81. D. Liu, B. Wang, H. Li, S. Huang, M. Liu et al., Distinguished $\mathrm{Zn}, \mathrm{Co}_{-}{ }_{\mathrm{N}}^{\mathrm{X}-\mathrm{C}-\mathrm{s}} \mathrm{y}$ active sites confined in dentric carbon for highly efficient oxygen reduction reaction and flexible $\mathrm{Zn}$-air Batteries. Nano Energy 58, 277-283 (2019). https://doi.org/ 10.1016/j.nanoen.2019.01.011

82. H. Li, L. Wang, Y. Dai, Z. Pu, Z. Lao et al., Synergetic interaction between neighbouring platinum monomers in $\mathrm{CO}_{2}$ hydrogenation. Nat. Nanotechnol. 13(5), 411-417 (2018). https://doi.org/10.1038/s41565-018-0089-z

83. S. Tian, Q. Fu, W. Chen, Q. Feng, Z. Chen et al., Carbon nitride supported $\mathrm{Fe}_{2}$ cluster catalysts with superior performance for alkene epoxidation. Nat. Commun. 9(1), 2353 (2018). https://doi.org/10.1038/s41467-018-04845-x

84. X. Meng, C. Ma, L. Jiang, R. Si, X. Meng et al., Distance synergy of $\mathrm{MoS}_{2}$-confined rhodium atoms for highly efficient hydrogen evolution. Angew. Chem. Int. Ed. 59(26), 1050210507 (2020). https://doi.org/10.1002/anie.202003484

85. P. Zhou, X. Hou, Y. Chao, W. Yang, W. Zhang et al., Synergetic interaction between neighboring platinum and ruthenium monomers boosts CO oxidation. Chem. Sci. 10(23), 5898-5905 (2019). https://doi.org/10.1039/c9sc00658c

86. Z. Lu, B. Wang, Y. Hu, W. Liu, Y. Zhao et al., An isolated zinc-cobalt atomic pair for highly active and durable oxygen reduction. Angew. Chem. Int. Ed. 58(9), 2622-2626 (2019). https://doi.org/10.1002/anie.201810175

87. X. Han, X. Ling, D. Yu, D. Xie, L. Li et al., Atomically dispersed binary Co-Ni sites in nitrogen-doped hollow carbon nanocubes for reversible oxygen reduction and evolution. Adv. Mater. 31(49), e1905622 (2019). https://doi.org/10. 1002/adma.201905622

88. L.M. Liu, N. Wang, C.Z. Zhu, X.N. Liu, Y.H. Zhu et al., Direct imaging of atomically dispersed molybdenum that enables location of aluminum in the framework of zeolite ZSM-5. Angew. Chem. Int. Ed. 59(2), 819-825 (2020). https://doi.org/10.1002/anie.201909834

89. H. Zhang, P. An, W. Zhou, B.Y. Guan, P. Zhang et al., Dynamic traction of lattice-confined platinum atoms into mesoporous carbon matrix for hydrogen evolution reaction. Sci. Adv. 4(1), eaao6657 (2018). https://doi.org/10.1126/sciadv.aao6657

90. X. Qiu, J. Chen, X. Zou, R. Fang, L. Chen et al., Encapsulation of C-N-decorated metal sub-nanoclusters/single atoms into a metal-organic framework for highly efficient catalysis. Chem. Sci. 9(48), 8962-8968 (2018). https://doi.org/10.1039/ c8sc03549k

91. S.H. Lee, J. Kim, D.Y. Chung, J.M. Yoo, H.S. Lee et al., Design principle of $\mathrm{Fe}-\mathrm{N}-\mathrm{C}$ electrocatalysts: How to optimize multimodal porous structures? J. Am. Chem. Soc. 141(5), 2035-2045 (2019). https://doi.org/10.1021/ jacs.8b11129

92. Q. Sun, N. Wang, T. Zhang, R. Bai, A. Mayoral et al., Zeolite-encaged single-atom rhodium catalysts: Highly-efficient hydrogen generation and shape-selective tandem hydrogenation of nitroarenes. Angew. Chem. Int. Ed. 58(51), 1857018576 (2019). https://doi.org/10.1002/anie.201912367

93. S. Ji, Y. Chen, S. Zhao, W. Chen, L. Shi et al., Atomically dispersed ruthenium species inside metal-organic frameworks: Combining the high activity of atomic sites and the molecular sieving effect of MOFs. Angew. Chem. Int. Ed. 58(13), 4271-4275 (2019). https://doi.org/10.1002/anie.201814182

94. R. Jiang, L. Li, T. Sheng, G. Hu, Y. Chen et al., Edge-site engineering of atomically dispersed $\mathrm{Fe}-\mathrm{N}_{4}$ by selective $\mathrm{C}-\mathrm{N}$ bond cleavage for enhanced oxygen reduction reaction activities. J. Am. Chem. Soc. 140(37), 11594-11598 (2018). https://doi.org/10.1021/jacs.8b07294

95. D. Liu, X. Li, S. Chen, H. Yan, C. Wang et al., Atomically dispersed platinum supported on curved carbon supports for efficient electrocatalytic hydrogen evolution. Nat. Energy 4(6), 512-518 (2019). https://doi.org/10.1038/s41560-019-0402-6

96. H. Zhang, W. Zhou, X.F. Lu, T. Chen, X.W. Lou, Implanting isolated $\mathrm{Ru}$ atoms into edge-rich carbon matrix for efficient electrocatalytic hydrogen evolution. Adv. Energy Mater. 10(23), 2000882 (2020). https://doi.org/10.1002/aenm.20200 0882

97. H. Zhang, S. Zuo, M. Qiu, S. Wang, Y. Zhang et al., Direct probing of atomically dispersed $\mathrm{Ru}$ species over multi-edged $\mathrm{TiO}_{2}$ for highly efficient photocatalytic hydrogen evolution. Sci. Adv. 6(39), eabb9823 (2020). https://doi.org/10.1126/ sciadv.abb9823

98. Z.Y. Li, Z. Yuan, X.N. Li, Y.X. Zhao, S.G. He, CO oxidation catalyzed by single gold atoms supported on aluminum oxide clusters. J. Am. Chem. Soc. 136(40), 14307-14313 (2014). https://doi.org/10.1021/ja508547z

99. R. Lin, D. Albani, E. Fako, S.K. Kaiser, O.V. Safonova et al., Design of single gold atoms on nitrogen-doped carbon for molecular recognition in alkyne semi-hydrogenation. Angew. Chem. Int. Ed. 58(2), 504-509 (2019). https://doi.org/10. 1002/anie.201805820

100. Z. Zhang, Y. Zhu, H. Asakura, B. Zhang, J. Zhang et al., Thermally stable single atom $\mathrm{Pt} / \mathrm{m}-\mathrm{Al}_{2} \mathrm{O}_{3}$ for selective hydrogenation and CO oxidation. Nat. Commun. 8, 16100 (2017). https://doi.org/10.1038/ncomms16100

101. X. Liu, M. Xu, L.Y. Wan, H.D. Zhu, K.X. Yao et al., Superior catalytic performance of atomically dispersed palladium on graphene in CO oxidation. ACS Catal. 10(5), 3084-3093 (2020). https://doi.org/10.1021/acscatal.9b04840

102. Y. Chen, J. Lin, L. Li, B. Qiao, J. Liu et al., Identifying size effects of Pt as single atoms and nanoparticles supported on $\mathrm{FeO}_{x}$ for the water-gas shift reaction. ACS Catal. 8(2), 859-868 (2018). https://doi.org/10.1021/acscatal.7b02751

103. Q. Shen, C. Cao, R. Huang, L. Zhu, X. Zhou et al., Single chromium atoms supported on titanium dioxide nanoparticles for synergic catalytic methane conversion under 
mild conditions. Angew. Chem. Int. Ed. 59(3), 1216-1219 (2020). https://doi.org/10.1002/anie.201913309

104. Y.X. Zhao, Z.Y. Li, Z. Yuan, X.N. Li, S.G. He, Thermal methane conversion to formaldehyde promoted by single platinum atoms in $\mathrm{PtAl}_{2} \mathrm{O}_{4}{ }^{-}$cluster anions. Angew. Chem. Int. Ed. 53(36), 9482-9486 (2014). https://doi.org/10.1002/ anie. 201403953

105. X. Guo, G. Fang, G. Li, H. Ma, H. Fan et al., Direct, nonoxidative conversion of methane to ethylene, aromatics, and hydrogen. Science 344(6184), 616-619 (2014). https://doi. org/10.1126/science. 1253150

106. P. Xie, T. Pu, A. Nie, S. Hwang, S.C. Purdy et al., Nanoceria-supported single-atom platinum catalysts for direct methane conversion. ACS Catal. 8(5), 4044-4048 (2018). https://doi.org/10.1021/acscatal.8b00004

107. M. Zhang, Y.G. Wang, W. Chen, J. Dong, L. Zheng et al., Metal (hydr)oxides@ polymer core-shell strategy to metal single-atom materials. J. Am. Chem. Soc. 139(32), 1097610979 (2017). https://doi.org/10.1021/jacs.7b05372

108. H. Zhou, Y. Zhao, J. Gan, J. Xu, Y. Wang et al., Cationexchange induced precise regulation of single copper site triggers room-temperature oxidation of benzene. J. Am. Chem. Soc. 142(29), 12643-12650 (2020). https://doi.org/ 10.1021/jacs.0c03415

109. S.W. Cao, H. Li, T. Tong, H.C. Chen, A.C. Yu et al., Singleatom engineering of directional charge transfer channels and active sites for photocatalytic hydrogen evolution. Adv. Funct. Mater. 28(32), 1802169 (2018). https://doi.org/10. 1002/adfm.201802169

110. Q. Zhao, J. Sun, S.C. Li, C.P. Huang, W.F. Yao et al., Single nickel atoms anchored on nitrogen-doped graphene as a highly active cocatalyst for photocatalytic $\mathrm{H}_{2}$ evolution. ACS Catal. 8(12), 11863-11874 (2018). https://doi.org/10. 1021/acscatal.8b03737

111. C. Gao, J. Low, R. Long, T. Kong, J. Zhu et al., Heterogeneous single-atom photocatalysts: Fundamentals and applications. Chem. Rev. 120(21), 12175-12216 (2020). https:// doi.org/10.1021/acs.chemrev.9b00840

112. Y. Pan, Y. Qian, X. Zheng, S.Q. Chu, Y. Yang et al., Precise fabrication oaf single-atom alloy co-catalyst with optimal charge state for enhanced photocatalysis. Natl. Sci. Rev. 8, nwaa224 (2021). https://doi.org/10.1093/nsr/nwaa224

113. J. Xing, J.F. Chen, Y.H. Li, W.T. Yuan, Y. Zhou et al., Stable isolated metal atoms as active sites for photocatalytic hydrogen evolution. Chem. Eur. J. 20(8), 2138-2144 (2014). https://doi.org/10.1002/chem.201303366

114. X. Li, W. Bi, L. Zhang, S. Tao, W. Chu et al., Single-atom $\mathrm{Pt}$ as co-catalyst for enhanced photocatalytic $\mathrm{H}_{2}$ evolution. Adv. Mater. 28(12), 2427-2431 (2016). https://doi.org/10. 1002/adma.201505281

115. X. Wu, H.B. Zhang, J.C. Dong, M. Qiu, J.T. Kong et al., Surface step decoration of isolated atom as electron pumping: Atomic-level insights into visible-light hydrogen evolution. Nano Energy 45, 109-117 (2018). https://doi.org/ 10.1016/j.nanoen.2017.12.039
116. J. Wang, T. Xia, L. Wang, X. Zheng, Z. Qi et al., Enabling visible-light-driven selective $\mathrm{CO}_{2}$ reduction by doping quantum dots: Trapping electrons and suppressing $\mathrm{H}_{2}$ evolution. Angew. Chem. Int. Ed. 57(50), 16447-16451 (2018). https:// doi.org/10.1002/anie.201810550

117. J. Di, C. Chen, S.Z. Yang, S. Chen, M. Duan et al., Isolated single atom cobalt in $\mathrm{Bi}_{3} \mathrm{O}_{4} \mathrm{Br}$ atomic layers to trigger efficient $\mathrm{CO}_{2}$ photoreduction. Nat. Commun. 10(1), 2840 (2019). https://doi.org/10.1038/s41467-019-10392-w

118. S. Ji, Y. Qu, T. Wang, Y. Chen, G. Wang et al., Rare-earth single erbium atoms for enhanced photocatalytic $\mathrm{CO}_{2}$ reduction. Angew. Chem. Int. Ed. 59(26), 10651-10657 (2020). https://doi.org/10.1002/anie.202003623

119. T. Chao, X. Luo, W. Chen, B. Jiang, J. Ge et al., Atomically dispersed copper-platinum dual sites alloyed with palladium nanorings catalyze the hydrogen evolution reaction. Angew. Chem. Int. Ed. 56(50), 16047-16051 (2017). https://doi.org/ 10.1002/anie. 201709803

120. M. Tavakkoli, N. Holmberg, R. Kronberg, H. Jiang, J. Sainio et al., Electrochemical activation of single-walled carbon nanotubes with pseudo-atomic-scale platinum for the hydrogen evolution reaction. ACS Catal. 7(5), 3121-3130 (2017). https://doi.org/10.1021/acscatal.7b00199

121. J. Zhang, Y. Zhao, X. Guo, C. Chen, C.-L. Dong et al., Single platinum atoms immobilized on an MXene as an efficient catalyst for the hydrogen evolution reaction. Nat. Catal. 1(12), 985-992 (2018). https://doi.org/10.1038/s41929-018-0195-1

122. K. Jiang, B. Liu, M. Luo, S. Ning, M. Peng et al., Single platinum atoms embedded in nanoporous cobalt selenide as electrocatalyst for accelerating hydrogen evolution reaction. Nat. Commun. 10(1), 1743 (2019). https://doi.org/10.1038/ s41467-019-09765-y

123. S. Fang, X. Zhu, X. Liu, J. Gu, W. Liu et al., Uncovering nearfree platinum single-atom dynamics during electrochemical hydrogen evolution reaction. Nat. Commun. 11(1), 1029 (2020). https://doi.org/10.1038/s41467-020-14848-2

124. J. Xu, C. Zhang, H. Liu, J. Sun, R. Xie et al., Amorphous $\mathrm{MoO}_{\mathrm{X}}$-stabilized single platinum atoms with ultrahigh mass activity for acidic hydrogen evolution. Nano Energy 70, 104529 (2020). https://doi.org/10.1016/j.nanoen.2020. 104529

125. L. Zhang, K. Doyle-Davis, X. Sun, Pt-based electrocatalysts with high atom utilization efficiency: from nanostructures to single atoms. Energy Environ. Sci. 12(2), 492-517 (2019). https://doi.org/10.1039/c8ee02939c

126. J. Deng, H. Li, J. Xiao, Y. Tu, D. Deng et al., Triggering the electrocatalytic hydrogen evolution activity of the inert two-dimensional $\mathrm{MoS}_{2}$ surface via single-atom metal doping. Energy Environ. Sci. 8(5), 1594-1601 (2015). https://doi.org/ $10.1039 / \mathrm{c} 5 \mathrm{ee} 00751 \mathrm{~h}$

127. Y. Xue, B. Huang, Y. Yi, Y. Guo, Z. Zuo et al., Anchoring zero valence single atoms of nickel and iron on graphdiyne for hydrogen evolution. Nat. Commun. 9(1), 1460 (2018). https://doi.org/10.1038/s41467-018-03896-4

128. H. Zhang, L. Yu, T. Chen, W. Zhou, X.W.D. Lou, Surface modulation of hierarchical $\mathrm{MoS}_{2}$ nanosheets by Ni single 
atoms for enhanced electrocatalytic hydrogen evolution. Adv. Funct. Mater. 28(51), 1807086 (2018). https://doi.org/ 10.1002/adfm.201807086

129. Y. Zhao, T. Ling, S. Chen, B. Jin, A. Vasileff et al., Non-metal single-iodine-atom electrocatalysts for the hydrogen evolution reaction. Angew. Chem. Int. Ed. 58(35), 12252-12257 (2019). https://doi.org/10.1002/anie.201905554

130. K. Qi, X. Cui, L. Gu, S. Yu, X. Fan et al., Single-atom cobalt array bound to distorted $1 \mathrm{~T} \mathrm{MoS}_{2}$ with ensemble effect for hydrogen evolution catalysis. Nat. Commun. 10(1), 5231 (2019). https://doi.org/10.1038/s41467-019-12997-7

131. H. Fei, J. Dong, M.J. Arellano-Jimenez, G. Ye, N. Dong Kim et al., Atomic cobalt on nitrogen-doped graphene for hydrogen generation. Nat. Commun. 6, 8668 (2015). https://doi. org/10.1038/ncomms9668

132. K. Chen, S. Kim, M. Je, H. Choi, Z. Shi et al., Ultrasonic plasma engineering toward facile synthesis of singleatom $\mathrm{M}-\mathrm{N}_{4} / \mathrm{N}$-doped carbon $(\mathrm{M}=\mathrm{Fe}, \mathrm{Co})$ as superior oxygen electrocatalyst in rechargeable zinc-air batteries. Nano-Micro Lett. 13, 60 (2021). https://doi.org/10.1007/ s40820-020-00581-4

133. B.S. Yeo, Oxygen evolution by stabilized single Ru atoms. Nat. Catal. 2(4), 284-285 (2019). https://doi.org/10.1038/ s41929-019-0271-1

134. Y. Hou, M. Qiu, M.G. Kim, P. Liu, G. Nam et al., Atomically dispersed nickel-nitrogen-sulfur species anchored on porous carbon nanosheets for efficient water oxidation. Nat. Commun. 10(1), 1392 (2019). https://doi.org/10.1038/ s41467-019-09394-5

135. Y. Li, Z.S. Wu, P. Lu, X. Wang, W. Liu et al., High-valence nickel single-atom catalysts coordinated to oxygen sites for extraordinarily activating oxygen evolution reaction. Adv. Sci. 7(5), 1903089 (2020). https://doi.org/10.1002/advs. 201903089

136. Q. Wang, X. Huang, Z.L. Zhao, M. Wang, B. Xiang et al., Ultrahigh-loading of Ir single atoms on $\mathrm{NiO}$ matrix to dramatically enhance oxygen evolution reaction. J. Am. Chem. Soc. 142(16), 7425-7433 (2020). https://doi.org/10.1021/ jacs.9b12642

137. W.J. Jiang, L. Gu, L. Li, Y. Zhang, X. Zhang et al., Understanding the high activity of $\mathrm{Fe}-\mathrm{N}-\mathrm{C}$ electrocatalysts in oxygen reduction: $\mathrm{Fe} / \mathrm{Fe}_{3} \mathrm{C}$ nanoparticles boost the activity of Fe-N ${ }_{x}$. J. Am. Chem. Soc. 138(10), 3570-3578 (2016). https://doi.org/10.1021/jacs.6b00757

138. C. Tang, Y. Jiao, B. Shi, J.N. Liu, Z. Xie et al., Coordination tunes selectivity: Two-electron oxygen reduction on highloading molybdenum single-atom catalysts. Angew. Chem. Int. Ed. 59(23), 9171-9176 (2020). https://doi.org/10.1002/ anie. 202003842

139. X. Luo, X. Wei, H. Wang, W. Gu, T. Kaneko et al., Secondary-atom-doping enables robust $\mathrm{Fe}-\mathrm{N}-\mathrm{C}$ singleatom catalysts with enhanced oxygen reduction reaction. Nano-Micro Lett. 12, 163 (2020). https://doi.org/10.1007/ s40820-020-00502-5

140. C.H. Choi, M. Kim, H.C. Kwon, S.J. Cho, S. Yun et al., Tuning selectivity of electrochemical reactions by atomically dispersed platinum catalyst. Nat. Commun. 7, 10922 (2016). https://doi.org/10.1038/ncomms10922

141. J. Liu, M. Jiao, L. Lu, H.M. Barkholtz, Y. Li et al., High performance platinum single atom electrocatalyst for oxygen reduction reaction. Nat. Commun. 8, 15938 (2017). https:// doi.org/10.1038/ncomms15938

142. S. Li, J. Liu, Z. Yin, P. Ren, L. Lin et al., Impact of the coordination environment on atomically dispersed Pt catalysts for oxygen reduction reaction. ACS Catal. 10(1), 907-913 (2019). https://doi.org/10.1021/acscatal.9b04558

143. J. Liu, M. Jiao, B. Mei, Y. Tong, Y. Li et al., Carbon-supported divacancy-anchored platinum single-atom electrocatalysts with superhigh Pt utilization for the oxygen reduction reaction. Angew. Chem. Int. Ed. 58(4), 1163-1167 (2019). https://doi.org/10.1002/anie.201812423

144. W.H. Lai, B.W. Zhang, Z. Hu, X.M. Qu, Y.X. Jiang et al., The quasi-Pt-allotrope catalyst: Hollow PtCo@single-atom $\mathrm{Pt}_{1}$ on nitrogen-doped carbon toward superior oxygen reduction. Adv. Funct. Mater. 29(13), 1807340 (2019). https://doi. org/10.1002/adfm.201807340

145. M. Xiao, J. Zhu, G. Li, N. Li, S. Li et al., A single-atom iridium heterogeneous catalyst in oxygen reduction reaction. Angew. Chem. Int. Ed. 58(28), 9640-9645 (2019). https:// doi.org/10.1002/anie.201905241

146. Z. Zhang, M. Dou, H. Liu, L. Dai, F. Wang, A facile route to bimetal and nitrogen-codoped 3D porous graphitic carbon networks for efficient oxygen reduction. Small 12(31), 4193-4199 (2016). https://doi.org/10.1002/smll.201601617

147. P. Chen, T. Zhou, L. Xing, K. Xu, Y. Tong et al., Atomically dispersed iron-nitrogen species as electrocatalysts for bifunctional oxygen evolution and reduction reactions. Angew. Chem. Int. Ed. 56(2), 610-614 (2017). https://doi. org/10.1002/anie.201610119

148. H.T. Chung, D.A. Cullen, D. Higgins, B.T. Sneed, E.F. Holby et al., Direct atomic-level insight into the active sites of a high-performance PGM-free ORR catalyst. Science 357(6350), 479-484 (2017). https://doi.org/10.1126/scien ce.aan 2255

149. P. Song, M. Luo, X.Z. Liu, W. Xing, W.L. Xu et al., Zn single atom catalyst for highly efficient oxygen reduction reaction. Adv. Funct. Mater. 27(28), 1700802 (2017). https://doi.org/ 10.1002/adfm.201700802

150. L. Jiao, G. Wan, R. Zhang, H. Zhou, S.H. Yu et al., From metal-organic frameworks to single-atom Fe implanted $\mathrm{N}$-doped porous carbons: Efficient oxygen reduction in both alkaline and acidic media. Angew. Chem. Int. Ed. 57(28), 8525-8529 (2018). https://doi.org/10.1002/anie.201803262

151. F. Li, G.-F. Han, H.-J. Noh, S.-J. Kim, Y. Lu et al., Boosting oxygen reduction catalysis with abundant copper single atom active sites. Energy Environ. Sci. 11(8), 2263-2269 (2018). https://doi.org/10.1039/c8ee01169a

152. C.C. Hou, L. Zou, L. Sun, K. Zhang, Z. Liu et al., Singleatom iron catalysts on overhang-eave carbon cages for highperformance oxygen reduction reaction. Angew. Chem. Int. Ed. 59(19), 7384-7389 (2020). https://doi.org/10.1002/anie. 202002665 
153. X. Wu, J.C. Dong, M. Qiu, Y. Li, Y.F. Zhang et al., Subnanometer iron clusters confined in a porous carbon matrix for highly efficient zinc-air batteries. Nanoscale Horiz. 5(2), 359-365 (2020). https://doi.org/10.1039/C9NH00510B

154. C.X. Zhao, B.Q. Li, J.N. Liu, Q. Zhang, Intrinsic electrocatalytic activity regulation of $\mathrm{M}-\mathrm{N}-\mathrm{C}$ single-atom catalysts for the oxygen reduction reaction. Angew. Chem. Int. Ed. 60(9), 4448-4463 (2020). https://doi.org/10.1002/anie.202003917

155. X. Chen, L. Yu, S. Wang, D. Deng, X. Bao, Highly active and stable single iron site confined in graphene nanosheets for oxygen reduction reaction. Nano Energy 32, 353-358 (2017). https://doi.org/10.1016/j.nanoen.2016.12.056

156. H. Zhang, W. Zhou, T. Chen, B.Y. Guan, Z. Li et al., A modular strategy for decorating isolated cobalt atoms into multichannel carbon matrix for electrocatalytic oxygen reduction. Energy Environ. Sci. 11(8), 1980-1984 (2018). https://doi. org/10.1039/c8ee00901e

157. T. Wang, X. Sang, W. Zheng, B. Yang, S. Yao et al., Gas diffusion strategy for inserting atomic iron sites into graphitized carbon supports for unusually high-efficient $\mathrm{CO}_{2}$ electroreduction and high-performance $\mathrm{Zn}-\mathrm{CO}_{2}$ batteries. Adv. Mater. 32(29), e2002430 (2020). https://doi.org/10.1002/adma. 202002430
158. W.Z. Zheng, F. Chen, Q. Zeng, Z.J. Li, B. Yang et al., A universal principle to accurately synthesize atomically dispersed metal- $\mathrm{N}_{4}$ sites for $\mathrm{CO}_{2}$ electroreduction. NanoMicro Lett. 12(1), 108 (2020). https://doi.org/10.1007/ s40820-020-00443-z

159. H. Zhang, W. Cheng, D. Luan, X.W. (David) Lou, Atomically dispersed reactive centers for electrocatalytic $\mathrm{CO}_{2}$ reduction and water splitting. Angew. Chem. Int. Ed. 60, 2-22 (2021). https://doi.org/10.1002/anie.202014112

160. Q. He, J.H. Lee, D. Liu, Y. Liu, Z. Lin et al., Accelerating $\mathrm{CO}_{2}$ electroreduction to $\mathrm{CO}$ over Pd single-atom catalyst. Adv. Funct. Mater. 30(17), 2000407 (2020). https://doi.org/ 10.1002/adfm.202000407

161. Y.N. Gong, L. Jiao, Y.Y. Qian, C.Y. Pan, L.R. Zheng et al., Regulating the coordination environment of MOF-templated single-atom nickel electrocatalysts for boosting $\mathrm{CO}_{2}$ reduction. Angew. Chem. Int. Ed. 59(7), 2705-2709 (2020). https:// doi.org/10.1002/anie.201914977

162. P. Huang, M. Cheng, H. Zhang, M. Zuo, C. Xiao et al., Single $\mathrm{Mo}$ atom realized enhanced $\mathrm{CO}_{2}$ electro-reduction into formate on $\mathrm{N}$-doped graphene. Nano Energy 61, 428-434 (2019). https://doi.org/10.1016/j.nanoen.2019.05.003 